\title{
Coherent level mixing in dot energy spectra measured by magnetoresonant tunneling spectroscopy of vertical quantum dot molecules
}

\author{
C. Payette,,${ }^{1,2, *}$ S. Amaha, ${ }^{3}$ G. Yu, ${ }^{1}$ J. A. Gupta, ${ }^{1}$ D. G. Austing,${ }^{1,2}$ S. V. Nair,${ }^{4}$ B. Partoens,${ }^{5}$ and S. Tarucha ${ }^{3,6}$ \\ ${ }^{1}$ Institute for Microstructural Sciences M50, National Research Council of Canada, Montreal Road, Ottawa, Ontario, Canada K1A OR6 \\ ${ }^{2}$ Department of Physics, McGill University, Ernest Rutherford Physics Building, 3600 rue University, \\ Montréal, Quebec, Canada H3A 2 T8 \\ ${ }^{3}$ ICORP Quantum Spin Information Project, Japan Science and Technology Agency, 3-1 Morinosato, Wakamiya, \\ Atsugi, Kanagawa 243-0198, Japan \\ ${ }^{4}$ Center for Advanced Nanotechnology, University of Toronto, 170 College Street, Toronto, Ontario, Canada M5S 3E3 \\ ${ }^{5}$ Department of Physics, University of Antwerp, Groenenborgerlaan 171, B-2020 Antwerp, Belgium \\ ${ }^{6}$ Department of Applied Physics, University of Tokyo, 7-3-1 Hongo, Bunkyo-ku, Tokyo 113-8656, Japan
}

(Received 17 February 2010; revised manuscript received 17 May 2010; published 10 June 2010)

\begin{abstract}
We study by magnetoresonant tunneling spectroscopy single-particle energy spectra of the constituent weakly coupled dots in vertical quantum dot molecules over a wide energy window. The measured energy spectra are well modeled by calculated spectra for dots with in-plane confinement potentials that are elliptical and parabolic in form. However, in the regions where two, three, or four single-particle energy levels are naively expected to cross, we observe pronounced level anticrossing behavior and strong variations in the resonant currents as a consequence of coherent mixing induced by small deviations in the nearly ideal dot confinement potentials. We present detailed analysis of the energy spectra, and focus on two examples of three-level crossings whereby the coherent mixing leads to concurrent suppression and enhancement of the resonant currents when the anticrossing levels are minimally separated. The suppression of resonant current is of particular interest since it is a signature of dark state formation due to destructive interference. We also describe in detail and compare two measurement strategies to reliably extract the resonant currents required to characterize the level mixing.
\end{abstract}

DOI: 10.1103/PhysRevB.81.245310

PACS number(s): 73.21.La, 73.22.Dj, 73.23.Hk

\section{INTRODUCTION}

Quantum dots (QDs) have attracted intense interest recently because they offer many exciting opportunities not only for exploring basic physics on the nanoscale but also for many new electronic and optical applications, including quantum information processing. ${ }^{1-7}$ Toward these goals, a concrete understanding of the basic properties of realistic QDs is actively being sought. The familiar Fock-Darwin spectrum, calculated nearly 80 years ago, shows the evolution of the energy of single-particle states for a strictly twodimensional circular parabolic confinement potential under the influence of an out-of-plane magnetic $(B)$ field. ${ }^{8,9}$ The Fock-Darwin states are widely used for the characterization and calculation of confined states in QD structures realized in a number of different ways. ${ }^{1-6}$ Since they possess a strong and well-defined confinement potential with a high degree of symmetry, ${ }^{10-13}$ the so-called vertical QDs are ideal vehicles for assessing to what degree in practice single-particle spectra of QD structures are Fock-Darwin-like in character over a wide energy window.

In this work, we describe detailed magnetoresonant tunneling spectroscopy measurements performed on vertical double-dot devices. From the measured single-particle spectra of two dots from two devices, we determine that the lateral confinement potentials of the dots are highly symmetric, and close to elliptical and parabolic in form. An initially unexpected, although positive, additional feature of the measured dot spectra is widespread anticrossing behavior and variation in the resonant currents when two, three and even four single-particle energy levels are brought into close proximity by the applied $B$ field. Recently, we took advantage of this feature as a means to study level anticrossing physics and quantum superposition phenomena (both of which are of broad interest in semiconducting nanosystems ${ }^{14}$ ), and demonstrated dark state formation due to destructive interference, witnessed by the complete suppression of an otherwise strong resonance, when three single-particle states are coherently mixed. ${ }^{15}$ This is an effect whose underlying physics is similar to that leading to coherent population trapping observed in quantum and atom optics. ${ }^{16,17}$ Here, we give extra details and methodologies relevant to the measurements of the energy spectra and the reliable characterization of the level mixing introduced in Ref. 15. Concerning the level mixing, emphasis is on three-level mixing, for which we provide an additional example of superposition leading to suppression of resonant current and thus the formation of a dark state.

This paper is organized in the following way. In Sec. II, we outline the principle of the measurement, provide layer details of the resonant-tunneling structure used, and include other general details about the device processing and measurements. In Sec. III, we describe the ideal calculated spectra most relevant to those measured and introduce the observed single-particle energy spectra of two dots. Unexpectedly, both of the measured spectra reveal pervasive and pronounced anticrossing behavior in the vicinity of the crossing points in the ideal calculated spectra. Additionally, we give an empirical procedure for estimating the dot confinement energies from the measured spectra. In Sec. IV, we 

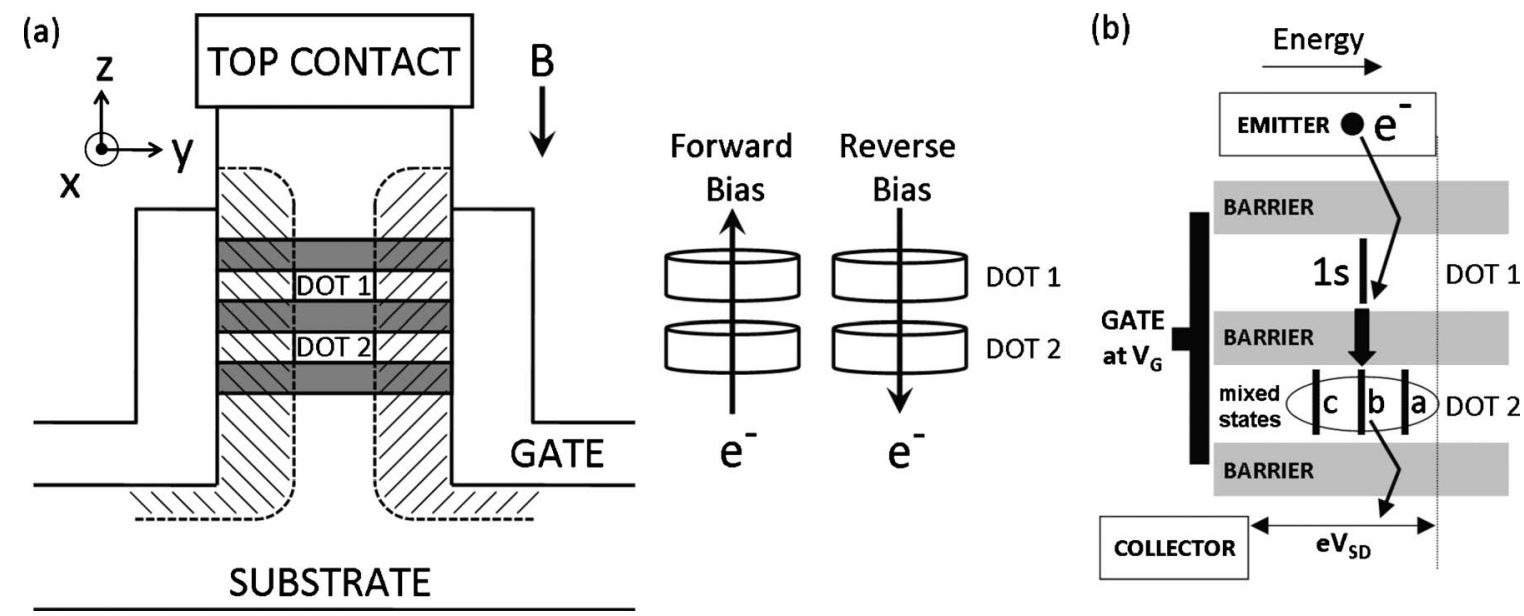

FIG. 1. (a) Schematic section of a vertical double-dot device also showing, using our bias convention, the direction of electron flow in forward and reverse bias, as well as the orientation of the applied $B$ field. (b) Cartoon of the measurement principle. Here, in reverse bias, with $V_{\mathrm{SD}}$ applied between the emitter and collector, the ground ( $1 s$-like) state of dot 1 probes three mixed states $a$, $b$, and $c$ in dot 2 (appropriate, for example, for the three-level mixing to be discussed in Sec. IV of the main text). The $1 s \rightarrow b$ resonance condition is shown and all empty nonresonant states at lower energy than $a, b$, and $c$ are omitted.

explain in detail how the measured energy spectra are captured and how we ensure that the spectral features we identify are single particle in nature. In Sec. V, we discuss and contrast two measurement strategies for reliably extracting the resonant currents at level crossings which, along with the $B$ field dispersion of the energy levels, contain valuable information about the coherent mixing. For illustration, we focus on a three-level crossing in the energy spectrum of one of the dots known to exhibit clear anticrossing behavior and strong suppression and enhancement of the resonant currents due to coherent mixing. ${ }^{15}$ In Sec. VI, we present, and analyze in depth, another example of a three-level crossing where these signatures of coherent mixing are also evident, and compare the behavior at this crossing with that under focus in Sec. V. The conditions leading to the strong suppression of resonant current and the formation of a dark state at these two crossings are explained. Section VII contains the conclusions and outlook.

\section{MEASUREMENT SCHEME AND DEVICE DETAILS}

For this study we use vertical double-dot devices [see Fig. 1(a) schematic]. The two weakly and vertically coupled dots are located in a submicron circular mesa with strong confinement in the vertical $(z)$ direction provided by heterostructure barriers [shaded dark gray in Fig. 1(a)]. The tunnel coupling in the $z$ direction between the two dots, $\Delta_{S A S}$, is sufficiently weak $(<0.1 \mathrm{meV})$ that it plays no significant role in our results and so we neglect it, i.e., we will take the states of interest to be the separate dot states and not bonding and antibonding states. ${ }^{18}$ The weaker confinement in the lateral $(x-y)$ direction is provided by sidewall depletion [regions with diagonal hatching in Fig. 1(a)] and can be tuned by applying a voltage $\left(V_{\mathrm{G}}\right)$ to a single metal gate wrapped around the mesa. In order to drive a current $(I)$ through the two dots in series, we apply a bias voltage between the top contact and the substrate contact $\left(V_{\mathrm{SD}}\right)$. We can induce cur- rent to flow through the device in either direction by applying positive or negative $V_{\mathrm{SD}}$. As shown in Fig. 1(a), we will refer to the situation of electrons flowing from the substrate contact to the top contact as forward bias and the opposite case as reverse bias. Defining dot 1 (dot 2) to be the QD nearest (furthest) from the top contact, our bias convention thus means that in forward (reverse) bias, electrons flow from the emitter contact through dot 2 (dot 1) first and then to dot 1 (dot 2) next before exiting to the collector contact. Furthermore, we apply a $B$ field in the $z$ direction, parallel to the tunneling current. As we will explain fully in Secs. III and IV, the goal is to map out, as a function of the applied $B$ field, the energy of the single-particle states of the downstream dot (furthest from the emitter) using the lowestenergy (ground) single-particle state of the upstream dot (nearest to the emitter) as a probe by covarying $V_{\mathrm{SD}}$ and $V_{\mathrm{G}}$ in order to bring single-particle energy levels into alignment and measure a resonant current [see also Fig. 1(b) which illustrates the measurement principle].

The devices are fabricated from a triple-barrier doublequantum-well resonant-tunneling structure grown on a heavily Si-doped GaAs substrate by molecular-beam epitaxy. The nominal layer details of the structure are as follows. First, four $n$-doped GaAs layers, designed to "step-down" the doping concentration, are deposited on top of the substrate. These layers have doping densities of $2 \times 10^{18}, 2 \times 10^{17}$, $1.4 \times 10^{17}$, and $1.2 \times 10^{17} \mathrm{~cm}^{-3}$ with thicknesses of $500 \mathrm{~nm}$, $180 \mathrm{~nm}, 150 \mathrm{~nm}$, and $70 \mathrm{~nm}$, respectively. These layers are followed by a $3 \mathrm{~nm}$ undoped GaAs spacer layer. Next are the layers which form the undoped central region of the triplebarrier double-quantum-well structure, namely, an $8.5 \mathrm{~nm}$ $\mathrm{Al}_{0.22} \mathrm{Ga}_{0.78} \mathrm{As}$ outer barrier, a $12 \mathrm{~nm} \mathrm{In}_{0.05} \mathrm{Ga}_{0.95} \mathrm{As}$ quantum well, an $8.5 \mathrm{~nm} \mathrm{Al}_{0.22} \mathrm{Ga}_{0.78} \mathrm{As}$ center barrier, a $12 \mathrm{~nm}$ $\mathrm{In}_{0.05} \mathrm{Ga}_{0.95} \mathrm{As}$ quantum well, and an $8.5 \mathrm{~nm} \mathrm{Al}_{0.22} \mathrm{Ga}_{0.78} \mathrm{As}$ outer barrier. These layers are followed by another $3 \mathrm{~nm}$ undoped GaAs spacer layer and then the doping concentration is "stepped-up" through four more doped GaAs layers. These layers have doping densities of $1.2 \times 10^{17}, 1.4 \times 10^{17}$, 
$2 \times 10^{17}$, and $2 \times 10^{18} \mathrm{~cm}^{-3}$ with thicknesses of $70 \mathrm{~nm}, 150$ $\mathrm{nm}, 180 \mathrm{~nm}$, and $17.5 \mathrm{~nm}$, respectively. In order to facilitate a nonalloyed ohmic contact to the top of the device mesa, ten $\delta$-doped layers of $\mathrm{Si}$ each with aerial density of 1.5 $\times 10^{13} \mathrm{~cm}^{-2}$ and each separated by $2.5 \mathrm{~nm}$ of GaAs doped to $2 \times 10^{18} \mathrm{~cm}^{-3}$ are then inserted. Finally, a GaAs layer 7-nmthick doped to $2 \times 10^{18} \mathrm{~cm}^{-3}$ is deposited forming the surface of the structure.

Throughout this work, we focus on measurements performed with two devices (referred to henceforth as device I and device II) which were both fabricated from the same structure described above. Device I (II) was processed and measured in Tokyo (Ottawa). All experiments on device I (II) were performed at $\sim 1.6 \mathrm{~K}(\sim 0.3 \mathrm{~K})$. The processing steps and other general details of the vertical double-dot devices have been described in depth elsewhere. ${ }^{19-21}$ The only substantial difference between devices I and II is that for device $\mathrm{I}$, a thin (nonconducting) line mesa is attached to the mesa containing the two coupled QDs while for device II no such line mesa is present. It is important to note that despite this, both device mesas are surrounded by a single gate and are nominally circular [device I (II) mesa diameter is $0.43 \mu \mathrm{m}$ $(0.55 \mu \mathrm{m})]$.

\section{CALCULATED AND MEASURED ENERGY SPECTRA}

The confined states in QD structures are commonly assumed to be those appropriate for a two-dimensional confinement potential that is strictly elliptical and parabolic in the $x-y$ plane, which is a reasonable starting point if the confinement in the $z$ direction is much stronger, as is the case for vertical QDs. ${ }^{22}$ Explicitly, the effective in-plane confinement potential is taken to be $V_{e f f}(x, y) \propto\left(\delta x^{2}+\delta^{-1} y^{2}\right)$, with ellipticity $\delta=\hbar \omega_{x} / \hbar \omega_{y} \geq 1$, where $\hbar \omega_{x}$ and $\hbar \omega_{y}$ are the confinement energies along the $x$ and $y$ axes, respectively.,23-26 We use the convention $\hbar \omega_{x} \geq \hbar \omega_{y}$ so that the major (minor) axis of the ellipse lies along the $y$ axis ( $x$ axis). If we consider only single-particle states and apply a $B$ field along the $z$ direction, this will result in the distinctive evolution of the energy of these states with $B$ field shown in the familiar Fock-Darwin spectrum ${ }^{8,9}$ when $\delta=1$ for a circular parabolic dot with confinement energy $\hbar \omega_{0}=\hbar \omega_{x}=\hbar \omega_{y}$. Spectra with clear and dominant Fock-Darwin-like characteristics have been observed in transport measurements of vertical QDs, ${ }^{10,12}$ and recently, for example, by magneto-optical spectroscopy of self-assembled InAs QDs, ${ }^{27}$ and by magnetotunneling spectroscopy of QDs induced in GaAs quantum wells by interstitial Mn ions. ${ }^{28}$

For an ideal circular parabolic confinement potential, the single-particle states can be labeled by two quantum numbers $(n, \ell)$ where $n$ and $\ell$ are, respectively, the radial quantum number and the orbital angular momentum quantum number. An equivalent and more commonly used notation is that of atomic orbitals, e.g., $1 s$ for the ground state in the first shell; $2 p^{+}$and $2 p^{-}$for the two states in the second shell and so on. The number in the atomic-orbital-like notation $M$ $=2 n+|\ell|+1$ indexes the shell and the $M$ th shell has $M$ states (degenerate at $0 \mathrm{~T}$ ). The letter in the atomic-orbital-like notation relates to $|\ell|$ with $|\ell|=0,1,2,3,4,5, \ldots$ corresponding to $s, p, d, f, g, h, \ldots$ while the + or - superscript refers to the sign of $\ell$. In the case of an ideal elliptical parabolic confinement potential since the orbital angular momentum quantum number is no longer appropriate, quantum numbers $n_{x}$ and $n_{y}$ are used instead of $n$ and $\ell$. Note that for $\delta=1,(n, \ell)$ can be converted to $\left(n_{x}, n_{y}\right)$ by the following relations: $n_{x}=n+\frac{1}{2}|\ell|$ $-\frac{1}{2} \ell$ and $n_{y}=n+\frac{1}{2}|\ell|+\frac{1}{2} \ell,{ }^{24}$ and now $M=n_{x}+n_{y}+1$. Even when the ellipticity is not unity, it is often less cumbersome and more transparent to label the states as if $\delta=1$, i.e., with the atomic-orbital-like notation.

Figure 2 shows two examples of ideal calculated singleparticle energy spectra, one for a circular parabolic confinement potential [Fig. 2(a)] and the other for an elliptical parabolic confinement potential [Fig. 2(b)]. The confinement energies selected for these spectra are appropriate for the measured energy spectra presented below. Furthermore, the spectra are plotted with the energy of the ground state subtracted from the energy of all states, which eliminates the influence of the diamagnetic shift of the ground state, as this is appropriate for the measurement scheme employed [see Fig. 1(b)], and hence for comparison with the measured spectra. While the lower part of the Fock-Darwin spectrum (up to the third shell) will likely be familiar to many, properties of the Fock-Darwin spectrum at higher energy (including the crossings between three or more levels), as well as the elliptical parabolic spectrum, are less well known. The Fock-Darwin spectrum and the elliptical parabolic spectrum are (strictly) for infinitely high two-dimensional confinement potentials (here, in the lateral direction) and so the energy levels in either spectrum can, in principle, be computed for arbitrarily high energies. ${ }^{29}$ Two-level crossings are clearly prevalent in the spectra, even starting from quite low down in energy, provided the $B$ field is not too large. However, to encounter level crossings between three or more energy levels at finite $B$ field, one must move progressively up in energy. For example, several three-level crossings and even a couple of four-level crossings are visible in the top half of the spectra in Fig. 2 for the energy range shown. In Ref. 15 and this work, we are primarily interested in the three-level crossings for detailed discussions of the coherent mixing, so, for convenience, the three lowest-energy three-level crossings are arbitrarily labeled $\tau, \gamma$, and $\pi$ in both spectra of Fig. 2.

The two ideal calculated spectra shown in Fig. 2 exhibit many similarities but also one striking difference and a second more subtle one. The most important similarity is that all the level crossings in both the ideal spectra are exact crossings, i.e., there is no anticrossing behavior between crossing single-particle energy levels. This is in contrast to the measured spectra to be discussed soon, where anticrossing behavior, attributed to anharmonicity and anisotropy, is prevalent. ${ }^{15}$ Another similarity is that the level crossings occur in "families" at certain distinct $B$ fields in these ideal spectra because the confinement energy is a constant for an entire spectrum. As an example, the $\tau$ and $\gamma$ crossings occur at the same $B$ field, along with many other level crossings, in both of the spectra. This too is different from what is observed in the measured spectra and will provide information about how the confinement energy itself changes with energy in practice. A further similarity between the two ideal spectra 
(a)

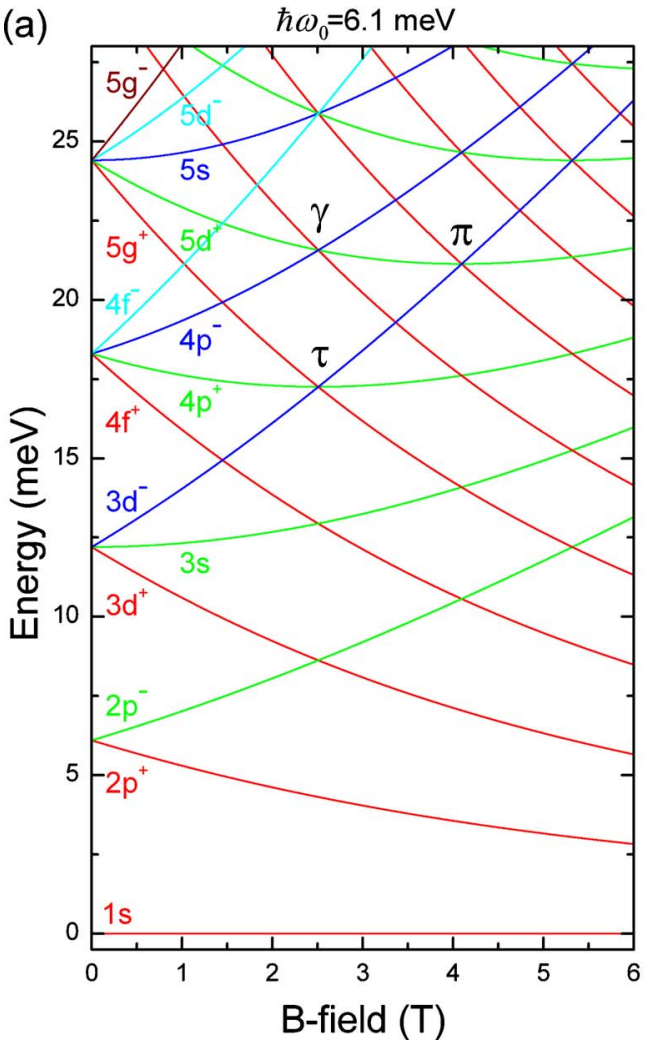

(b)

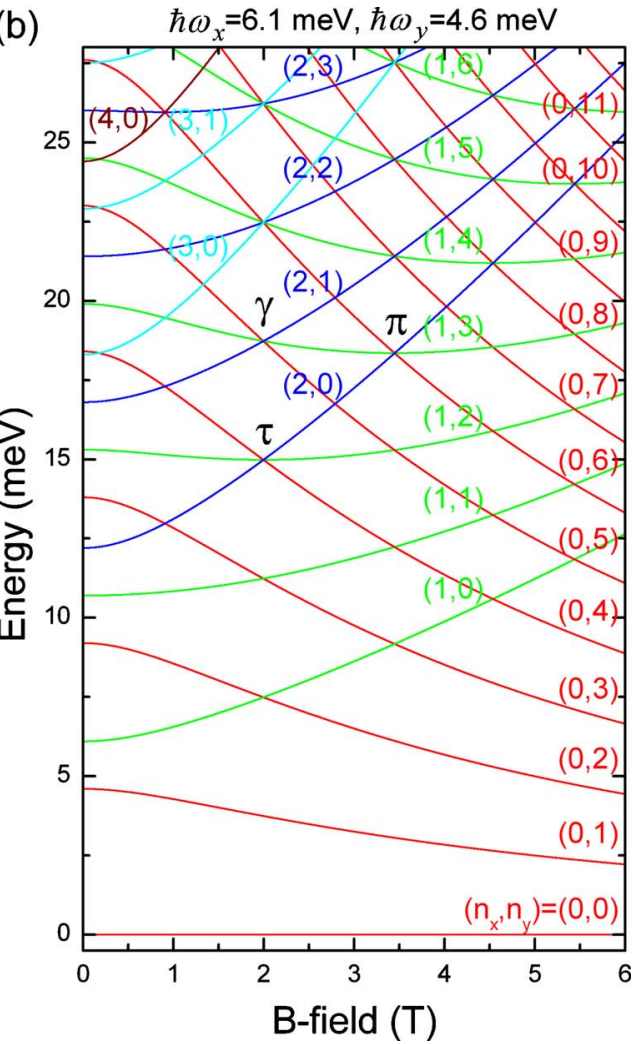

FIG. 2. (Color online) (a) Fock-Darwin spectrum calculated with $\hbar \omega_{0}=6.1 \mathrm{meV}$. The states originating from the first five shells at $0 \mathrm{~T}$ are labeled with their familiar atomic-orbital-like notation. (b) Single-particle energy spectrum of an elliptical parabolic dot calculated with $\hbar \omega_{x}=6.1 \mathrm{meV}$ and $\hbar \omega_{y}=4.6 \mathrm{meV}$. Here, the states are labeled using the $n_{x}$ and $n_{y}$ quantum numbers. In both (a) and (b), energy levels which merge into the same Landau level at high $B$ field are colored the same and energy of all states is with respect to the energy of the ground state, i.e., the diamagnetic shift of the ground state has been subtracted from all energy levels. The lowest three-level crossings are labeled $\gamma, \tau$, and $\pi$ in each spectrum.

is that at very high $B$ field, well beyond $6 \mathrm{~T}$, the energy levels with the same $n_{x}$ quantum number merge into the same Landau level. At such $B$ fields, the influence of an ellipticity greater than unity diminishes because the cyclotron orbits become much smaller then those arising solely from the lateral confinement imposed by the sidewall depletion. The most striking difference between the two ideal spectra is the general lifting of the degeneracies of the energy levels in the same shell at $0 \mathrm{~T}$ that is introduced when $\delta>1$. In this situation, another more subtle difference depends on the size and precise value of $\delta$. To see this, consider, for example, the two-level crossing between the $2 p^{-}$and $3 d^{+}$states which, when $\delta=1$, occurs at finite $B$ field. As $\delta$ is increased from unity [with the constraint that $\left(\hbar \omega_{x}\right)\left(\hbar \omega_{y}\right)$ is constant], the crossing between the two related states $\left[\left(n_{x}, n_{y}\right)=(1,0)\right.$ and $(0,2)]$ shifts progressively to lower $B$ field. Eventually, at $\delta$ $=2$, the crossing occurs at $0 \mathrm{~T}$, and for $\delta>2$ it will not take place at all. Generalizing, if $\delta$ becomes too large, level crossings will disappear, particularly in the lower part of the energy spectrum, and one would need to go to ever higher energy to encounter the level crossings. Also note that for certain "magic" values of $\delta$ ( $\delta=2$ is one example), widespread level degeneracy will occur at $0 \mathrm{~T}$ between levels which cross at finite $B$ field when $\delta=1 .{ }^{24,26}$ For the situation most relevant for the measured spectra to be introduced next $\delta \leq 1.5$. In this case, the ideal circular parabolic and the el- liptical parabolic single-particle spectra are quite similar in form for $B>1 \mathrm{~T}$, as is clear from Fig. 2.

Consistent with the measurement scheme stated in Sec. II [see also Fig. 1(b)], in the single-electron (elastic) resonanttunneling regime, we can measure the single-particle energy spectrum of the downstream dot by using the $1 s$-like state of the upstream dot as a probe (energy filter). ${ }^{12}$ Energy spectra (mapping out directly the position of current peaks as explained in Secs. IV and V) of two dots are shown in Fig. 3. The spectrum in Figs. 3(a) and 3(b) reveals levels which are almost degenerate within shells at $0 \mathrm{~T}$ indicating that the first probed dot (dot 2 from device I) is circular to a high degree. In contrast, for the spectrum in Figs. 3(c) and 3(d) the levels are now well separated at $0 \mathrm{~T}$, indicating that the second probed dot (dot 2 from device II) has higher ellipticity. ${ }^{30}$ Two further key observations are readily apparent on examination of the measured spectra. First, an attractive attribute of acquiring energy spectra in the way we do with a vertical double-dot device is that we can easily access the singleparticle states over a large energy window, limited only by the onset of longitudinal-optic-phonon emission at $\sim 37 \mathrm{meV} .{ }^{31}$ This window is much larger than that possible with "current stripe" measurements on a single vertical QD, where excited states of the one-electron system can only be clearly observed if they lie within a $\sim 5 \mathrm{meV}$ window (limited by $\left.\hbar \omega_{0}\right) .{ }^{11}$ Second, and most importantly for the coher- 

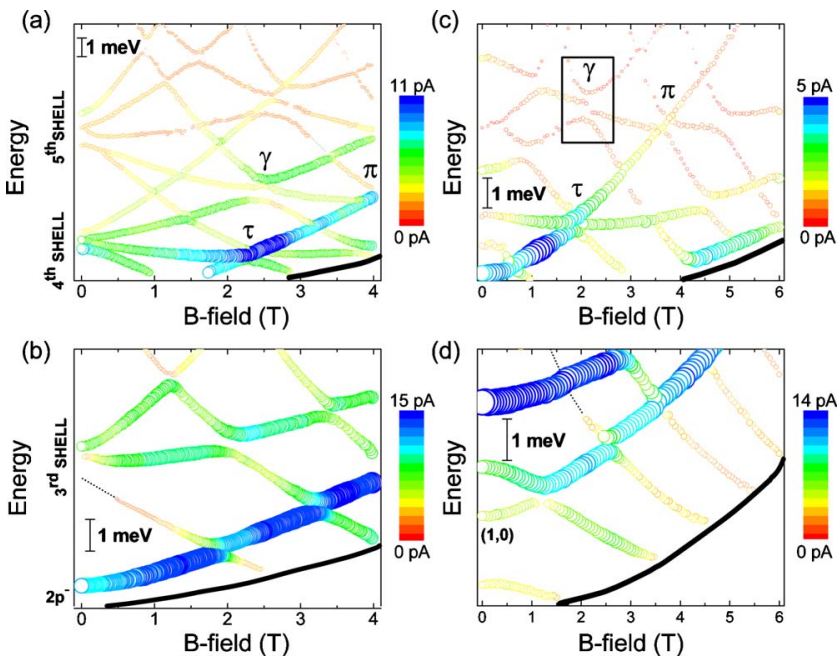

FIG. 3. (Color online) Energy spectrum of dot 2 from device I [(a) and (b)], and energy spectrum of dot 2 from device II [(c) and (d)] both of which reveal widespread level mixing. The reason why there are upper and lower parts to each spectrum is explained in Sec. IV of the main text. The approximately picoampere resonant current (nonresonant background current not removed) is shown. Dotted black lines identify barely resolvable portions of some weak spectral features. The energy scales are estimated by comparison to ideal calculated spectra (see Sec. III of the main text). The $2 p^{-}$-like state and the related $\left(n_{x}, n_{y}\right)=(1,0)$-like state are marked in (b) and (d), respectively. The thick black lines at the bottom right of each panel are explained in Sec. IV of the main text.

ent level mixing of interest, the naive expectation from the ideal spectra of Fig. 2 that all the level crossings should be exact is manifestly incorrect as we observe widespread anticrossing behavior in Fig. 3 when two or more single-particle energy levels approach each other. Many two-level crossings show pronounced anticrossing behavior (see Refs. 21 and 32 for a basic introduction), as do the three lowest-energy threelevel crossings labeled $\tau, \gamma$, and $\pi$ (as outlined in Ref. 15 for the spectrum of dot 2 from device II). Interestingly, in Fig. 3(a), just above the $\gamma$ crossing, a clear four-level crossing exhibiting anticrossing behavior is also visible. ${ }^{33}$ We have argued previously that this anticrossing behavior can arise from natural perturbations in the confinement potential of real QDs caused by local randomness and imperfections, ${ }^{15,30}$ and that by including appropriate symmetry-breaking terms in the confinement potential of the downstream dot, we can explain our experimental observations at specific crossings. ${ }^{34}$

The strength of the spectral features in Fig. 3 reflects the resonant current and this too can provide valuable information about the nature of transport through the QDs. If the two dots are assumed to be both ideal and identical, one might expect that only the $1 s \rightarrow 1 s$ resonance should be observed for our measurement scheme due to wave-function orthogonality, i.e., the $1 s \rightarrow X$ resonant current would be zero if $X$ is any downstream dot state other than $1 s .{ }^{12}$ In actuality, we find that almost all $1 s \rightarrow X$ resonances carry nonzero current (of order $1-10 \mathrm{pA}$ ) indicating that there is a finite tunnel coupling between the upstream dot's $1 s$-like state and the downstream dot's probed states which we attribute to the symmetry-breaking perturbations in the dot confinement potentials ${ }^{15,18,34}$ Evident from the spectra presented in Fig. 3, these currents vary nontrivially from resonance-to-resonance and as a function of $B$ field. We note that in the original work of Ono et al., ${ }^{12}$ two possible pictures for the resonant currents were put forward: coherent tunneling and (incoherent) sequential tunneling. While recently we have successfully applied the coherent tunneling picture to explain pronounced level mixing effects, ${ }^{15,34}$ like those presented later in Secs. V and VI, for selected crossings, a full microscopic model that can explain a sizeable portion of the spectrum (energy and current) does not yet exist.

Neglecting the mixing of levels in the regions where the levels are anticipated to cross exactly, overall the measured energy spectrum of dot 2 from device I shown in Figs. 3(a) and 3(b) is well reproduced by the calculated Fock-Darwin spectrum shown in Fig. 2(a), and likewise overall the measured energy spectrum of dot 2 from device II shown in Figs. $3(\mathrm{c})$ and 3(d) is well reproduced by the calculated elliptical parabolic spectrum shown in Fig. 2(b). The fact that the agreement is so good justifies, consistent with the measurement scheme [see also discussion in Sec. IV], the subtraction of the energy of the ground state from the energy of all higher states in the calculated spectra shown in Fig. 2. Indeed, had we not done this subtraction, the agreement would be not quite so good since the energy levels in the calculated spectra would, at any given $B$ field, all have larger gradients than actually observed.

In order to estimate the confinement energies of the probed dots from the measured spectra in Fig. 3 (required for the calculated spectra in Fig. 2), we note that for an ideal elliptical parabolic potential, both the relative spacing of the energy levels at $0 \mathrm{~T}$ and the $B$ field position of the exact level crossing points are uniquely determined by the confinement energies $\hbar \omega_{x}$ and $\hbar \omega_{y}$. Utilizing this principle, we can take a measured spectrum and estimate the confinement energies by matching it with a calculated elliptical parabolic spectrum. This then allows us to set the energy scale bars in Fig. 3. What the "energy" axis actually corresponds to in the measurements will be explained in Sec. IV. In the case of the energy spectrum of dot 2 from device I shown in Figs. 3(a) and $3(\mathrm{~b})$, an estimate reveals $\delta \approx 1.05$, which is sufficiently close to unity that we will treat this dot as being circular in the following discussions. ${ }^{10}$ This is quite reasonable since the splitting of the levels in the shells at $0 \mathrm{~T}$ is small compared to the energy separation between the shells, e.g., see the nearly degenerate four (five) levels in the fourth (fifth) shell in Fig. 3 (a). In contrast, for dot 2 from device II $\delta$ is estimated from Figs. 3(c) and 3(d) to be $\sim 1.3-1.5$ (see confinement energies quoted below) so this probed dot evidently cannot be treated as being approximately circular.

In practice, there is one complication in determining the confinement energies of the probed dots from the measured spectra that cannot be neglected. Recalling that the energylevel crossings in the ideal spectra calculated for fixed confinement occur in families of crossings at certain distinct $B$ fields, close inspection of the measured spectra reveals that higher-energy crossings in any given "family" are in fact systematically shifted to higher $B$ field. For instance, in the spectrum of dot 2 from device II [Fig. 3(c)], we see that the (centers of the) $\tau$ and $\gamma$ crossings occur, respectively, at 


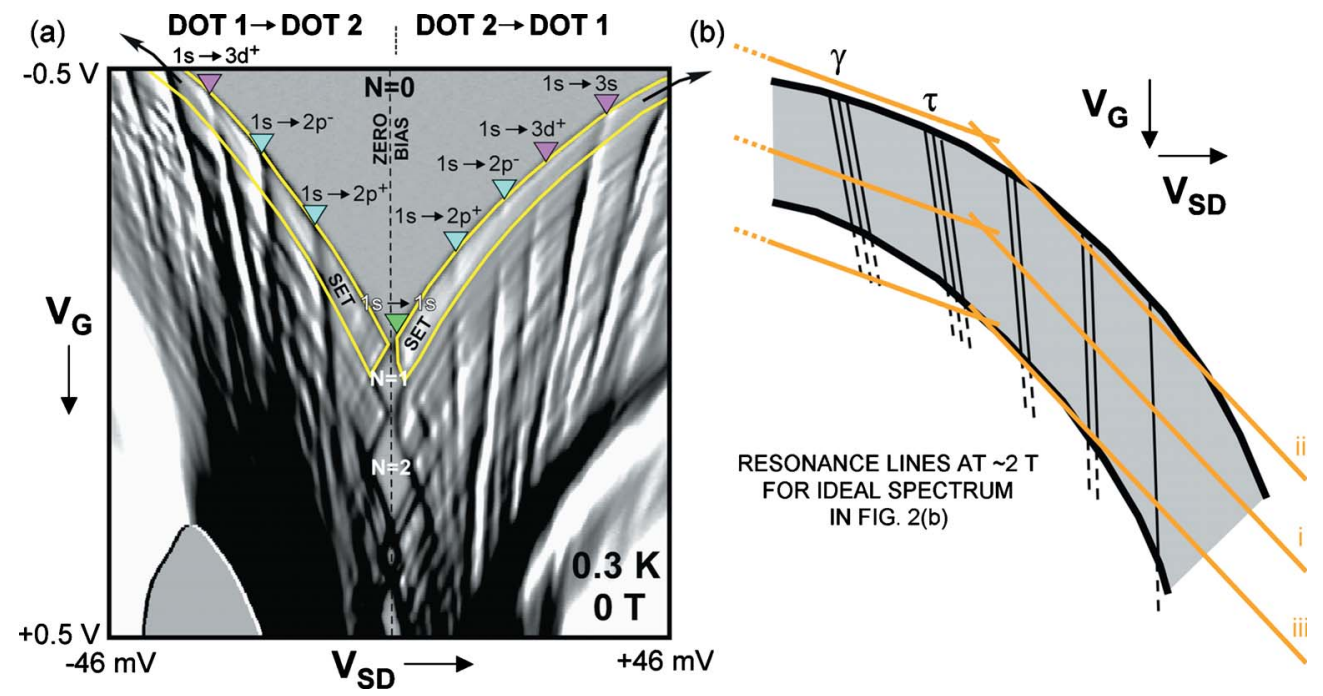

FIG. 4. (Color online) (a) Differential conductance, $d I / d V_{\mathrm{SD}}$, in the $V_{\mathrm{SD}}-V_{\mathrm{G}}$ plane for device II at 0 T. Black, gray, and white, respectively, represents positive, zero, and negative conductance. The current is less than $200 \mathrm{pA}$ (except in the gray region in the lower left of the figure). The $N=1$ and 2 Coulomb diamonds are marked, and the two arc-shaped regions of primary interest are highlighted and marked SET. The dot 1 (dot 2) spectrum can be measured in forward (reverse) bias. The first few resonance lines involving the lower energy levels of the probed dot are marked by triangles (and labeled with the atomic-orbital-like notation for simplicity although the dots' spectra are elliptical in nature). (b) Cartoon of extended reverse bias SET region (in gray) showing three possible two-part vector-voltage line scans numbered i, ii, and iii [see Sec. IV in the main text for discussion]. The situation depicted, with the spectrum of dot 2 from device II in mind [see Figs. 3(c) and 3(d)], is of the nearly vertical resonance lines cut at $\sim 2 \mathrm{~T}$ if the dot 2 spectrum were that shown in Fig. 2(b). For this condition, the resonance lines relevant to the $\tau$ and $\gamma$ crossings are minimally separated. When the resonance lines pass below the SET region into the region where other tunneling processes can additionally occur, they are shown as dashed lines, and not all resonance lines that may appear in this region are shown (Ref. 39).

$\sim 1.9 \mathrm{~T}$ and $\sim 2.2 \mathrm{~T}$. The reason for this, which will also become clearer in Sec. IV, is that in our measurement scheme, in order to capture a spectrum at higher energy, more negative $V_{\mathrm{G}}$ is required, and hence the dots are "squeezed" more. ${ }^{35}$ Thus, the effective confinement of the probed dot actually increases along the energy axis of a spectrum which explains why the members of a family of crossings are not all observed at the same $B$ field as naively expected from the ideal calculated spectra of Fig. 2. Consequently, we chose to determine what are essentially average confinement energies separately for each panel in Fig. 3. To do this, we selected a low-energy crossing point and a high-energy crossing point in each panel, which would both occur at the same $B$ field if the confinement were constant, took the average of their actual $B$ field positions, and then used this to generate the appropriate calculated spectrum. The choice of confinement energies in Fig. 2 is now clear. In Fig. 2(a), we show the Fock-Darwin spectrum calculated with a confinement energy of $\hbar \omega_{0}=6.1 \mathrm{meV}$, which is appropriate for the upper part of the spectrum of dot 2 from device I [Fig. 3(a)] while the lower part of this dot's spectrum [Fig. 3(b)] is best modeled by a Fock-Darwin spectrum calculated with $\hbar \omega_{0}=4.8 \mathrm{meV}$ (not shown). As a useful rule-of-thumb, we note that the confinement energy can be estimated from a Fock-Darwin spectrum by noting the $B$ field (in tesla) at which the lowest-energy two-level crossing (between the $2 p^{-}$and $3 d^{+}$states) occurs and multiplying this by 2.47 to obtain the confinement energy in millielectron volt. In Fig. 2(b), we show an elliptical parabolic spectrum calculated with confinement energies $\hbar \omega_{x}=6.1 \mathrm{meV}$ and $\hbar \omega_{y}=4.6 \mathrm{meV}$, i.e., $\delta \approx 1.33$, which is appropriate for the upper part of the spectrum of dot 2 from device II [Fig. 3(c)] while the lower part of this dot's spectrum [Fig. 3(d)] is best modeled by an elliptical parabolic spectrum calculated with $\hbar \omega_{x}=5.4 \mathrm{meV}$ and $\hbar \omega_{y}=3.5 \mathrm{meV}$, i.e., $\delta \approx 1.54$ (not shown).

\section{ENERGY SPECTRA MEASUREMENTS}

Although the nature of the spectra in Fig. 3 is apparent, how the spectra are actually measured will be less familiar, and so we now describe this in detail with the aid of a specific example (see also Ref. 21 for a basic introduction to the measurement techniques). Figure 4(a) shows the differential conductance in the $V_{\mathrm{SD}}-V_{\mathrm{G}}$ plane for device II at $0 \mathrm{~T}$. Right at the top of the gray $\mathrm{V}$-shaped region, the device is "pinchedoff" and there are no electrons trapped in either dot (marked " $N=0 ")$. At zero bias, on moving toward more positive $V_{\mathrm{G}}$, we see the first few Coulomb (blockade) diamonds, where the number of electrons $(N)$ trapped in the two-dot system is constant. Also identified are the two arc-shaped regions of primary interest in which sequential single-electron tunneling (SET) through the two dots occurs in the absence of electrons being permanently trapped on either dot. ${ }^{12,21,32,36}$ Below these two SET regions, at more positive $V_{\mathrm{G}}$ and away from the Coulomb diamonds at low bias, transport can take place by double, triple, quadruple,... electron tunneling. ${ }^{37}$ Features due to higher-order tunneling through just the downstream dot are present in the gray V-shaped region just above the SET regions but here, for the chosen gray scale, 
they are too weak to discern $(I<50 \mathrm{fA})$. We note that in Ref. 36, the center barrier was thinner ( $6 \mathrm{~nm}$ compared to 8.5 $\mathrm{nm}$ in our devices), and so these features, although still weak, were easier to see.

In order to capture the energy spectrum of one of the dots in device II, we measure the current, at fixed $B$ field, by scanning along straight "vector-voltage" lines (ideally solely) within one of the arc-shaped SET regions shown in Fig. 4(a) and then step the $B$ field. Along these vector-voltage lines, which constitute the energy axes in the measured spectra, $V_{\mathrm{SD}}$ and $V_{\mathrm{G}}$ are changed colinearly so that we can cut across the resonance lines of interest, the nearly vertical white-black lines in Fig. 4(a) inside the SET region (marked by colored triangles) in either bias direction in the $V_{\mathrm{SD}^{-}} V_{\mathrm{G}}$ plane. ${ }^{38}$ This leads to a series of current peaks (the maximum of each peak is located at the boundary between the white region and the black region of each of the white-black lines) whose positions change as a function of $B$ field, and thus an energy spectrum is built up. The SET regions in the $V_{\mathrm{SD}^{-}} V_{\mathrm{G}}$ plane extend well beyond the highlighted regions in Fig. 4(a), in the direction of the black arrows, and due to their extent and shape it is not possible to measure an energy spectrum over a very large energy window with a single straight vectorvoltage line scan. Thus, we usually measure a spectrum in at least two parts, as is the case in Fig. 3 for both devices, i.e., one vector-voltage line scan captures the lower part of the spectrum at lower bias and another vector-voltage line scan, of different gradient, captures a higher part of the spectrum at higher bias [see, for example, scan i in Fig. 4(b)]. When setting up a vector-voltage line scan, the aim is to ensure that the line always remains inside the target SET region. However, the resonances may not be visible if all or part of the vector-voltage line is above the SET region and instead cuts into the V-shaped region of zero current in Fig. 4(a) [see, for example, scan ii in Fig. 4(b)]. Another scenario is that all or part of the vector-voltage line cuts into the region just below the SET region possibly resulting in extra spectral features that arise only when permanent dot charging occurs [see, for example, scan iii in Fig. 4(b)]. If this occurs it is straightforward to exclude the unwanted extra resonance lines by comparison with the calculated spectra, and so we are confident that the spectra in Fig. 3 are single particle in nature. ${ }^{15,39} \mathrm{~A}$ further complication is that due to the above-mentioned diamagnetic shift of a dot's ground state, both the SET regions systematically move down, toward more positive $V_{\mathrm{G}}$, relative to any fixed vector-voltage line with increasing $B$ field. Because of this shift, it can be challenging to set up the vectorvoltage line scan appropriately so that it stays substantially within the target SET region over the course of an entire measurement (typically over a 4 or $6 \mathrm{~T}$ range). As a final comment on the vector-voltage line scans, we note that for convenient reference we normally start the vector-voltage line scan in the V-shaped region of zero current but still close to the target SET region. This explains why the measured parts of the spectra only "appear" above the thick black line at the bottom right of each of the panels in Fig. 3.

Each resonance line inside the SET regions of Fig. 4(a) corresponds to a single-particle state in the downstream dot being probed by the $1 s$-like (ground) state of the upstream dot [for instance, the $1 s \rightarrow 2 p^{+}, 1 s \rightarrow 2 p^{-}$, and $1 s \rightarrow 3 d^{+}$re- verse bias resonance lines are identified on the left side of Fig. 4(a)]. The measured reverse bias spectrum shown in Figs. 3(c) and 3(d) for device II actually corresponds to the spectrum of dot 2 being probed by the $1 s$-like state of dot 1 [see also cartoon in Fig. 1(b)]. The lower part of the spectrum displayed in Fig. 3(d) is essentially captured with a vector-voltage line scan through the reverse bias SET region shown in Fig. 4(a) (although the $1 s \rightarrow 2 p^{+}$resonance line is no longer cut by the vector-voltage line above $\sim 1.5 \mathrm{~T}) .{ }^{39}$ The upper part of the spectrum displayed in Fig. 3(c) is captured with another vector-voltage line scan through the same reverse bias SET region but at more negative $V_{\mathrm{SD}}$ and $V_{\mathrm{G}}{ }^{39}$ Furthermore, we note that resonance lines are also visible on the forward bias side of Fig. 4(a), where the dot being probed (dot 1) is the dot which provided the $1 s$-like "prober" state for the reverse bias spectrum in Figs. 3(c) and 3(d). Spectra of both dots in a device may thus be measured. Depending on the nature of the perturbations in the confinement potentials of the dots, these spectra may or may not be similar in appearance. $^{40}$

We note that the $1 s \rightarrow 1 s$ resonance line in Fig. 4(a) does not occur exactly at zero bias as one might anticipate because of a small energy mismatch between the two dots in device II. ${ }^{41}$ Nonetheless, the fact that the $1 s \rightarrow 1 s$ resonance line still occurs quite close to zero bias (just to the right, in forward bias) indicates that the ladders of single-particle energy levels in the two dots are almost aligned at zero bias, which explains why the Coulomb diamonds are still well formed. ${ }^{41-43}$ This contrasts with the case where the dots have a larger energy mismatch at zero bias, in which case the Coulomb diamonds appear less well formed and not to close. Such a situation is usefully encountered in the study of spinblockade physics. ${ }^{36}$ Since for device II, the (only) $1 s \rightarrow 1 s$ resonance line occurs in forward bias, it is not possible to probe the ground state of dot 2 with any vector-voltage line that lies solely in reverse bias.

The fact that the spectral lines in Fig. 3 are well reproduced by the ideal calculated spectra in Fig. 2 (away from the immediate crossing regions) confirms their singleparticle nature and the involvement of only the $1 s$-like state of the upstream dot. ${ }^{44}$ Were other higher-energy states in the upstream dot available for tunneling, or electrons permanently trapped on either dot, we would expect the spectral lines shown in Fig. 3 to be very different. Given the clear nature of the measured spectra, apparently the $1 s$-like (ground) state of the upstream dot is "pinned" close to the Fermi level of the emitter, even for the typically high bias voltages applied to capture the single-particle spectra [up to $\sim 150 \mathrm{mV}$, for example, in Fig. 3(a)], indicating that the electric field across the downstream dot is much larger. Beyond the scope of the current work, it would be interesting if a suitably sophisticated and realistic self-consistent calculation with our device parameters could reproduce our empirical observations at high bias.

\section{STRATEGIES FOR EXTRACTING RESONANT CURRENTS AT LEVEL CROSSINGS}

We now turn our attention from the "global" properties of an energy spectrum to those of individual crossings in a 
spectrum. Mixing of single-particle energy levels due to symmetry-breaking perturbations in the confinement potential of the downstream dot can explain why anticrossing behavior is commonly observed, rather than the anticipated exact crossing, whenever two or more single-particle energy levels are brought into proximity with an applied $B$ field. ${ }^{15,34}$ However, key information demonstrating directly the coherent nature of the mixing is also present in the $B$ field dependence of the resonant currents and hence, reliable means to extract the resonant currents at a crossing region are essential for the in-depth modeling of this mixing.

We have developed two measurement strategies, which we refer to as $\mathrm{A}$ and $\mathrm{B}$, for determining the resonant currents at a crossing region. Strategy $A$ is to measure the energy versus $B$ field spectrum with vector-voltage line scans, as discussed in the previous section, and extract the resonant currents directly in the vicinity of the crossing of interest. Strategy B is to measure the resonance lines inside one of the arc-shaped SET regions of the $V_{\mathrm{SD}^{-}} V_{\mathrm{G}}$ plane for different fixed $B$ fields [similar to Fig. 4(a) but in one bias direction only] and then to determine the current from different points along each resonance line for a specific crossing.

As a concrete example to illustrate the two measurement strategies, for the remainder of this section, we focus on one three-level crossing: the $\gamma$ crossing between the $4 p^{-}-, 5 d^{+}$, and $6 h^{+}$-like single-particle states in the spectrum of dot 2 from device II [see boxed region in Fig. 3(c)]. For this crossing, we previously demonstrated that coherent mixing of the states strongly affects the resonant current for each of the resulting branches and even leads to the complete suppression of the resonant current in one of the branches. ${ }^{15}$ The numerically derived differential conductance ${ }^{38}$ plot for the $\gamma$ crossing is presented in Fig. 5(a). The data shown is from the same data set used to build up the spectrum in Fig. 3(c). Figure 5(b) shows explicitly the branch resonances in every second one of the current traces measured in the vicinity of the $\gamma$ crossing. In both panels, we clearly see three distinct branches, which we refer to as the upper, center, and lower branches [identified, respectively, by the symbols $\triangle, \bigcirc$, and $\nabla]$. Starting from the left of the crossing region, the weak (dark) upper and lower branch resonances approach the strong (bright) center branch resonance as the $B$ field is increased. At the center of the crossing region (near $\sim 2.2 \mathrm{~T}$ ), when the branches are minimally separated, the upper and lower branch resonances have become strong (bright) while the center branch resonance has been completely suppressed (becomes "dark"). As the $B$ field is increased further, the center branch resonance recovers its strength and the other two branch resonances start to weaken.

Even "on-resonance," not all of the measured current is solely resonant current and so the resonant current component must be extracted for each of the relevant current peaks from the vector-voltage line scans acquired in strategy $\mathrm{A}$ measurements. To do this, we begin by carefully subtracting the non-negligible nonresonant background current from each of the current traces [like those shown in Fig. 5(b) but usually a wider range in energy is necessary in order to identify, fit and then remove the background current correctly]. This background current is $\sim 0.2 \mathrm{pA}$ in the vicinity of the $\gamma$ crossing, and although it fluctuates a little from trace-to- (a)

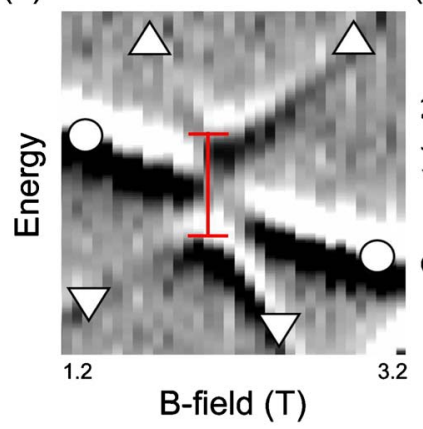

(c)

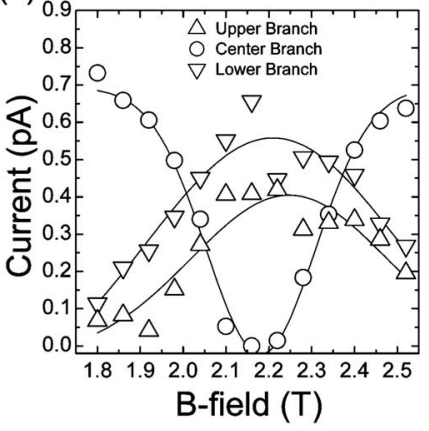

(b)

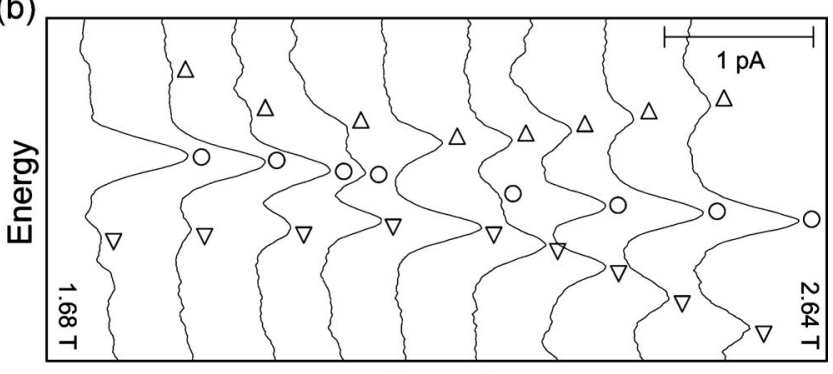

Current

FIG. 5. (Color online) (a) Energy-level [(differential conductance resonance (Ref. 38)] position versus $B$ field for the three-level crossing $\gamma$ from the spectrum of dot 2 from device II [see boxed region in Fig. 3(c)]. Black, gray, and white, respectively, represents positive, zero, and negative conductance. The energy scale bar corresponds to $\sim 0.8 \mathrm{meV}$, and the upper, center, and lower branches, respectively, are labeled by the symbols $\triangle, \bigcirc$, and $\nabla$. (b) Selected (smoothed) current traces which when numerically differentiated form vertical sections of the plot in (a) (nonresonant background current not removed). The peaks of the three resonances are also marked by $\triangle, \bigcirc$, and $\nabla$ for each trace, except where the peaks are too weak to identify. (c) Current values (resonant current with nonresonant background component subtracted) versus $B$ field for each branch extracted by strategy A from the current traces used to build up the plot in (a). The black lines are a guide to the eyes (and are generated by simple Gaussian fitting although no meaning is attached to the fitting procedure).

trace, a quadratic fit is found to be sufficient to account for the generally smooth and weak variation along each trace, and so usually the background current can be easily removed. Note that if we did not properly remove the background current the true nature of the coherent mixing would be obscured. Once the background current is subtracted, we find that a simultaneous fit of the current peaks of interest with unconstrained Lorentzians is sufficient to extract the resonant current component for each current peak. The result of this procedure for the three branches of the $\gamma$ crossing is given in Fig. 5(c). The $B$ field dependence of the branch currents is now clearly revealed and reflects well the behavior discussed qualitatively above for Figs. 5(a) and 5(b), namely, the bright resonance-to-dark resonance interconversion on tuning the $B$ field through the crossing. ${ }^{15}$

Appealingly, the single-particle nature of the spectrum is directly apparent in a strategy A measurement without detailed knowledge of how it is acquired (and the measurement is relatively quick in comparison with a strategy B measure- 
ment, as we will discuss below). However, recalling the practical points related to setting up a good vector-voltage line scan discussed in Sec. IV [see also Fig. 4(b)], there are some further notes of caution. Foremost, each resonance is a line within a SET region. Thus, even if we can ensure that the vector-voltage line scan always remains inside the target SET region, so excluding the possibility of picking up unwanted extra spectral features, it only cuts through each of the resonance lines of interest at one point, i.e., the choice of a good vector-voltage line is not unique. Furthermore, the position of the point (relative to say the lower and upper edges of the SET region) where the fixed vector-voltage line cuts each resonance line will inevitably change during a spectral measurement as the diamagnetic shift effect causes the SET region to move toward more positive $V_{\mathrm{G}}$ with increasing $B$ field. Because of these factors, we considered it possible that the precise position of the points where the vector-voltage line cuts the resonance lines could significantly alter the appearance of the crossing and, in particular, the behavior of the branch currents, so leading to a completely different interpretation of the underlying coherent mixing. For example, would the center branch resonance of the $\gamma$ crossing still "vanish" at the center of the crossing if the vector-voltage line scans were set up differently?

Because of these concerns, we originally employed strategy $B$, which we will now describe fully, to extract the resonant current for the branches at the $\gamma$ crossing. ${ }^{15}$ Figure 6 shows the differential conductance in the relevant region of the $V_{\mathrm{SD}}-V_{\mathrm{G}}$ plane in the vicinity of the $\gamma$ crossing at eight different $B$ fields. The same general behavior evident in Fig. 5(a) is clearly seen again in Fig. 6, namely, three distinct resonance lines on the low $(1.8-2.0 \mathrm{~T})$ and high $(2.4-2.5 \mathrm{~T})$ $B$ field side of the crossing, and when the resonance lines are minimally separated, at $2.2 \mathrm{~T}$, only two resonance lines (the upper and lower branches) are visible since the center resonance line (the center branch) has been completely suppressed. Interestingly, in the $2.1 \mathrm{~T}$ and $2.3 \mathrm{~T}$ panels, only part of the center resonance line (upper part at $2.1 \mathrm{~T}$ and lower part at $2.3 \mathrm{~T}$ ) is clearly visible. That all or part of the center resonance line is absent only for a narrow $B$ field range demonstrates that the presence of the dark state at the center of the crossing region observed in the strategy A measurement (see Fig. 5) is not critically sensitive to the choice of vectorvoltage line. In the panels of Fig. 6, although not yet understood, we do see some small variations (short range with respect to $V_{\mathrm{G}}$ ) in the strength and width of the white-black resonance lines on moving from the lower edge to the upper edge of the region of interest in which SET dominates. ${ }^{45,46}$ Nonetheless, in order to track any systematic variation (long range with respect to $V_{\mathrm{G}}$ ) along the length of the resonance lines which might influence our interpretation of the underlying mixing, we determine the value of the resonant current at points a quarter, half, and three quarters of the way along each of the three resonance lines inside the region of interest (see $2.4 \mathrm{~T}$ panel of Fig. 6 for definition of these points ${ }^{45}$ ), for different $B$ fields close to the crossing region. Figures 7(a)-7(c) show the extracted resonant currents, with the nonresonant background current subtracted, for each of these points. Although the background current could, in principle, depend on three parameters, namely, $B$ field, $V_{\mathrm{G}}$ and $V_{\mathrm{SD}}$, we

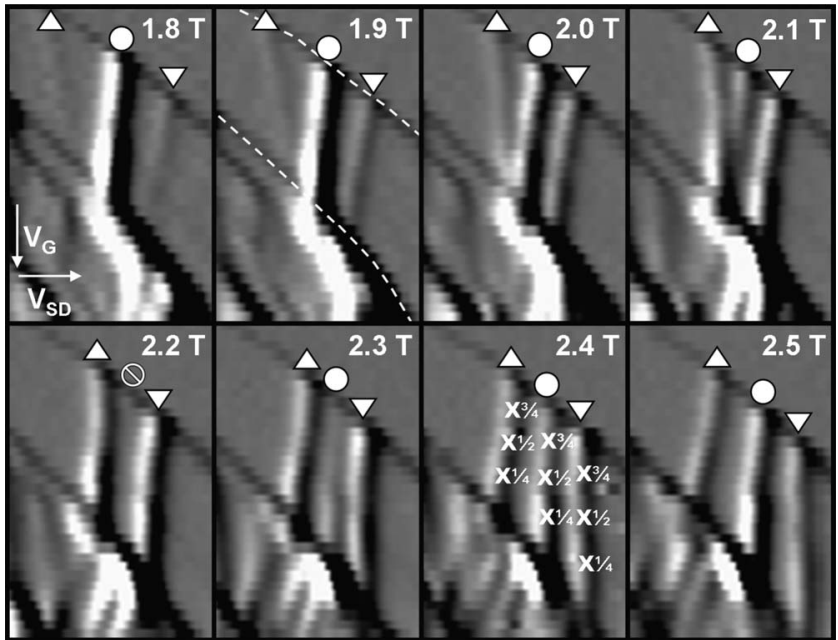

FIG. 6. Panels showing gray scale plots of the differential conductance, $d I / d V_{\mathrm{SD}}$, in the relevant region of the $V_{\mathrm{SD}}-V_{\mathrm{G}}$ plane in the vicinity of the $\gamma$ crossing in the spectrum of dot 2 from device II at eight different $B$ fields. The gray scale is in the same sense as that in Fig. 4(a). Dashed lines in the 1.9 T panel highlight the lower and upper edges of the region of interest in which SET dominates (Ref. 45). In this region, the upper, center, and lower branch resonance lines, respectively, are labeled by the symbols $\Delta, \bigcirc$, and $\nabla$. Due to the extent and shape of the SET region, the time required for the measurements is minimized by capturing data from a parallelogram-shaped region in the $V_{\mathrm{SD}^{-}} V_{\mathrm{G}}$ plane rather than a (more conventional) rectangular-shaped region [as was done in Fig. 4(a)]. However, the data is displayed here in rectangular-shaped panels for which the start and end points of the $V_{\mathrm{SD}}$ sweep are systematically shifted together for each value of $V_{\mathrm{G}}$ [stepped from -0.55 to $-0.9 \mathrm{~V}$ from bottom to top] such that at the bottom (top) of each panel, $V_{\mathrm{SD}}=-47 \mathrm{mV}(-100 \mathrm{mV})$ on the left side and $V_{\mathrm{SD}}=-31 \mathrm{mV}(-84 \mathrm{mV})$ on the right side of the panel. The crosses in the $2.4 \mathrm{~T}$ panel are explained in Sec. $\mathrm{V}$ of the main text in connection with Fig. 7.

find that only the $V_{\mathrm{G}}$ dependence is significant, although even its dependence is comparatively weak in the vicinity of the $\gamma$ crossing. In detail, the background current is examined along two series of points parallel to the resonance lines, one series to the left of the resonance line marked by $\triangle$ and the other series to the right of the resonance line marked by $\nabla$, at two fixed $B$ fields $(2.0 \mathrm{~T}$ and $2.3 \mathrm{~T})$, and a quadratic fit is found to be sufficient to account for the $V_{\mathrm{G}}$ dependence. At $2.1 \mathrm{~T}$, the lower part of the center resonance line is not visible and so the corresponding quarter-point resonant current has been set to zero in Fig. 7(a). Lastly, in Fig. 7(d) we show for each $B$ field, the average values of the currents at the three points for each branch, and we note that it is essentially this average which was shown and modeled in Ref. 15.

Figures 7(a)-7(c) can be viewed as approximately mimicking the outcome of three reasonable vector-voltage line scans which could have been used in strategy A measurements, i.e., line scans which cut across all three resonance lines and have slopes appropriate for capturing a reasonably large portion of the dot's single-particle energy spectrum. Evidently, the $B$ field dependences of the branch currents at the three selected points along the resonance lines shown in 
(a)

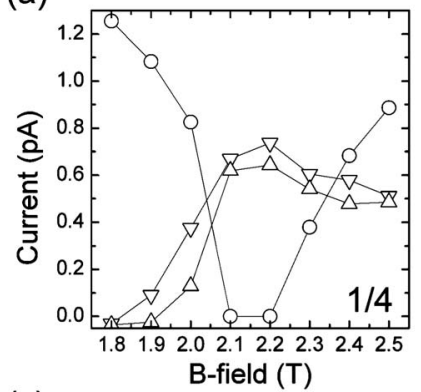

(c)

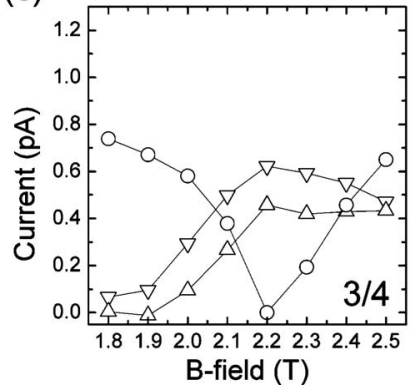

(b)

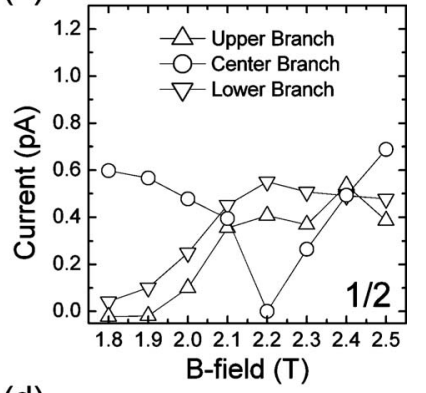

(d)

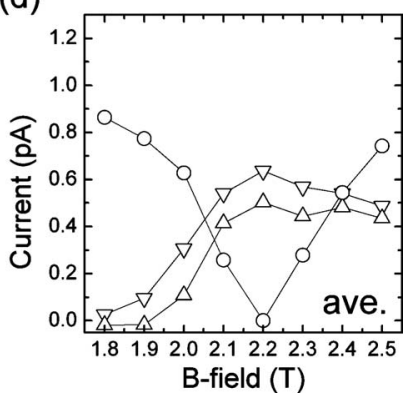

FIG. 7. Current values (resonant current with nonresonant background component subtracted) versus $B$ field for each branch of the $\gamma$ crossing in the spectrum of dot 2 from device II extracted by strategy B at points (a) a quarter, (b) half, and (c) three quarters of the way along each of the three resonance lines inside the region of interest [see 2.4 T panel of Fig. 6 for definition of these points (Ref. 45)]. The average values of the currents at these three points are shown in (d).

Figs. $7(a)-7(c)$ are qualitatively the same. This reveals that the overall behavior of the branch currents, and so the interpretation of the underlying physics, is essentially independent of the detailed choice of the position of the point along each of the resonance lines at which the current is determined provided the choice is sensible, i.e., the relative position of the point should be approximately the same for all the resonance lines. Hence, this provides strong evidence that when using strategy A it does not depend critically where the (straight) vector-voltage line cuts the resonance lines of interest so long as they are all sensibly cut inside the region where SET dominates. ${ }^{45}$ Using either measurement strategy, it is clear in all respects that the following is robust: to the low and high $B$ field side of the crossing, the upper and lower branch resonances are weak and the center branch resonance is strong while at the center of the crossing, the upper and lower branch resonances are strong and the center branch resonance is completely suppressed. Quantitatively, the measured current values for each branch do vary in a fairly systematic fashion with position along the resonance lines [see Figs. 7(a)-7(c)] with the current of each branch generally decreasing on moving from the lower edge to the upper edge of the region of interest. Reassuringly, when we compare the branch currents obtained using strategy A [Fig. 5(c)] with those obtained from strategy B (Fig. 7), we see that at any given $B$ field current values for the former lie within the range of the current values for the latter. Most likely, the vector-voltage line scan used in the strategy A measurement cut the resonance lines somewhere near the half and three

quarter points. ${ }^{45}$ Finally, we note that the noise level in our measurements of the current with either strategy is estimated to be $\sim 25$ fA.

To summarize this section we compare the advantages and disadvantages of the two measurement strategies. The main advantage of using strategy $\mathrm{A}$ is that it takes significantly less time to acquire a high-resolution spectrum than using strategy B (hitherto not mentioned but see upcoming comments). Nonetheless, strategy A is more difficult to implement as setting up an appropriate vector-voltage line scan for the entire $B$ field range of interest can be challenging due to, for instance, the diamagnetic shift effect discussed in Sec. IV. To be quantitative, a typical strategy A measurement lasts $\sim 15 \mathrm{~h}$. For instance, the spectral features shown in Fig. 3(c) [Fig. 3(d)] came from approximately one half (one third) of a single strategy A measurement. On the other hand, with strategy B it would have taken $\sim 500 \mathrm{~h}$ to build up a comparable part of the spectrum to that shown in Figs. 3(c) and 3(d) and involved measurements similar to that shown in Fig. 4(a) but for only one bias direction and repeated over the $0 \mathrm{~T}$ to $6 \mathrm{~T}$ range in steps of $0.06 \mathrm{~T}$. Although feasible, not only would the measurement of the spectrum by strategy B be exceedingly long, it would be tedious to assemble the data sets to generate the spectrum. One could justifiably ask if it is possible to attempt to measure only a specific crossing, by either strategy, with the goal of increasing the resolution in mind [the $\gamma$ crossing data actually shown in Figs. 5(a) and 6 is only a small fraction of the full data sets measured]. However, attempting to measure only one particular crossing would certainly be impossible without having first examined at least one extensive $V_{\mathrm{SD}}-V_{\mathrm{G}}$ differential conductance plot at $0 \mathrm{~T}$ [like that shown in Fig. 4(a)] to gain the necessary information in order to set up sensible vector-voltage line scans. Next, the single-particle spectrum of one of the dots must be measured over a sufficiently large energy and $B$ field range, like that shown in Figs. 3(c) and 3(d), in order to locate the target crossing. Furthermore, even then, a highresolution measurement of only a specific crossing would still be challenging as without a larger measurement window identifying, features may be difficult to locate, and determining the nonresonant background current accurately may prove impossible in the case of strategy A. The main advantage of strategy B is that it provides significantly more information by measuring whole resonance lines in the SET region (or the extended region in which SET dominates ${ }^{45}$ ), although it will also provide extensive information about the area outside the region of interest. However, due to the additional time required to capture large data sets from an extensive region in the $V_{\mathrm{SD}^{-}}-V_{\mathrm{G}}$ plane for each $B$ field, the $B$ field resolution of the strategy $\mathrm{B}$ measurements is in practice reduced compared with the strategy A measurements, e.g., the $B$ field step size in Fig. 6 is $0.1 \mathrm{~T}$ while it is $0.06 \mathrm{~T}$ for Fig. 5(a). Nonetheless, regardless of the measurement strategy employed, the $\gamma$ crossing reveals, in particular, a dramatic suppression of the otherwise strong center branch resonance when the $B$ field is tuned to the center of the crossing. In Ref. 15, with the model to be discussed in the next section, we established that this behavior is a strong signature of coherent mixing leading to destructive interference and thus dark state formation when two of the three possible cou- 


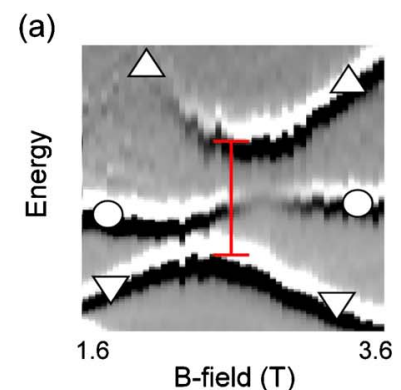

(c)

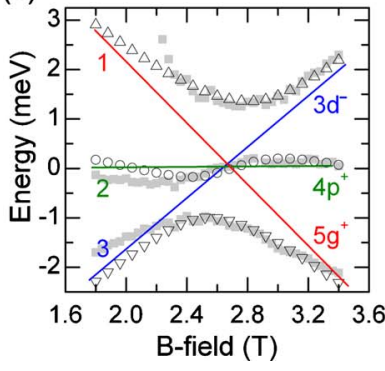

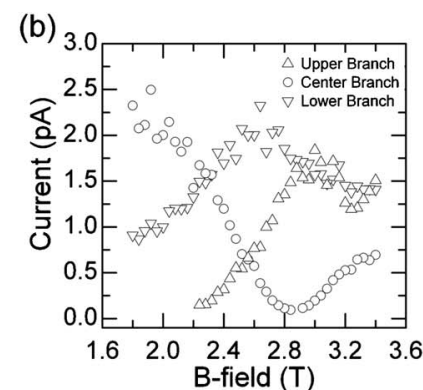

(d)

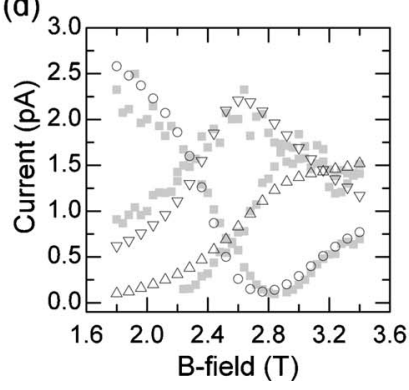

(e) $1.0 \quad$ Upper Branch
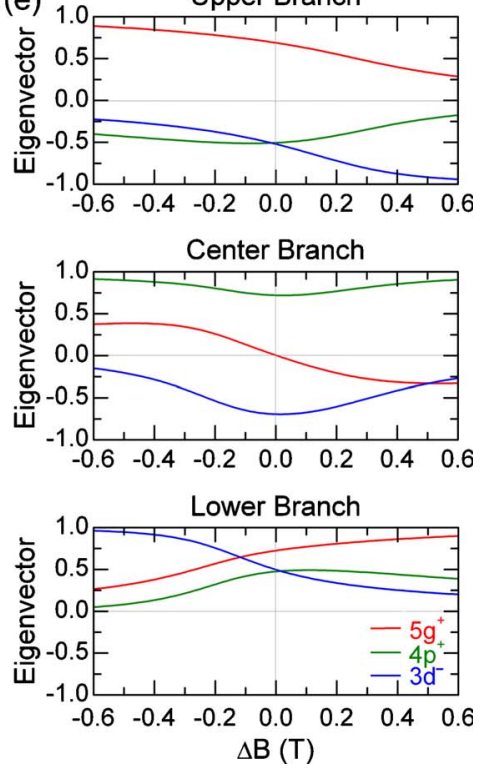

FIG. 8. (Color online) (a) Energy-level [differential conductance resonance (Ref. 38)] position versus $B$ field for the three-level crossing $\tau$ from the spectrum of dot 1 from device I [not shown (Ref. 40)]. The grayscale is in the same sense as that in Fig. 5(a). The energy scale bar corresponds to (a surprisingly large) $\sim 2.5 \mathrm{meV}$, and the upper, center, and lower branches, respectively, are labeled by the symbols $\triangle$, $\bigcirc$, and $\nabla$. (b) Current values (resonant current with nonresonant background component subtracted) versus $B$ field for each branch extracted by strategy A from the current traces used to build up the plot in (a). The upper branch current to the left of $\sim 2.2 \mathrm{~T}$ is too small to determine. (c) Fit of energy-level positions. The colored lines estimating the position of the uncoupled basis levels provide a guide to the eyes. The energy of the point where the three uncoupled basis levels cross is set to zero energy. The energy-level position data extracted from (a) is also included (gray squares). (d) Fit of branch currents. The data points from (b) are also included (gray squares). (e) Reconstructed eigenvectors of the three branches showing the components of the uncoupled basis states on passing through the crossing region. $\Delta B=0 \mathrm{~T}$ is at $\sim 2.68 \mathrm{~T}$.

plings between the basis states in the downstream dot are dominant and the other coupling is much weaker.

\section{ANALYSIS OF COHERENT MIXING AT A THREE-LEVEL CROSSING}

Figures 8(a) and 8(b) show the behavior at another threelevel crossing: the $\tau$ crossing between the $3 d^{-}-, 4 p^{+}-$, and $5 g^{+}$-like single-particle states in the spectrum of dot 1 from device I [the bias direction is opposite to that of the measured spectrum in Figs. 3(a) and 3(b)]. Like the $\gamma$ crossing from the spectrum of dot 2 from device II discussed in the preceding section, this crossing too has three distinct branches. Furthermore, the center branch resonance is also strongly suppressed near the center of the crossing region when the branches are minimally separated, and we will argue below that this is another example of dark state formation by coherent three-level mixing. Although at first sight, the behavior at the $\tau$ crossing appears quite similar to that at the $\gamma$ crossing in Fig. 5, the $\tau$ crossing does exhibit some subtle differences as compared to the $\gamma$ crossing. For instance, the center of the $\tau$ crossing occurs at $\sim 2.68 \mathrm{~T}$ [see Fig. 8(c)] while the center branch current is most strongly suppressed at $\sim 2.86 \mathrm{~T}$ [as shown in Fig. 8(b)], i.e., the suppression minimum is shifted to the right of the center of the crossing region. Additionally, there is also a pronounced "wiggle" in the energy-level position of the center branch evident in Fig. 8(a). Furthermore, although there is enhancement of the resonant current, the upper and lower branch currents are nonetheless dissimilar with the former (latter only) showing a monotonic increase (a maximum) close to the center of the crossing [see Fig. 8(b)].

In order to quantitatively discuss the mixing, we first recap the simple model, introduced in Ref. 15, with which we can compute and fit both the energy-level position and the resonant current for each of the three branches throughout any three-level crossing region. The first part of the model consists of a $3 \times 3$ matrix Hamiltonian that allows us to study mixing at a generic level crossing between three approaching and initially uncoupled basis levels, each assumed to have a linear dispersion with $B$ field. For ease of discussion (and generality), we label these basis levels (states) 1, 2, and 3 in the sense indicated in Fig. 8(c). Specifically for the $\tau$ crossing, basis states 1,2 , and 3 correspond, respectively, to the $5 g^{+}-, 4 p^{+}$, and $3 d^{-}$-like states. We note that in general, the energy levels in the ideal calculated and measured spectra have nonlinear $B$ field dispersion but over a limited $B$ field range near a crossing point, a linear dispersion is a reasonable approximation. Three off-diagonal matrix elements, the coupling energy parameters $C_{12}, C_{13}$, and $C_{23}$, characterize the couplings between each pair of basis states. They are assumed to be real, but may be positive or negative, and independent of $B$ field and of each other. In the second part of the model, current amplitude parameters $s_{1}, s_{2}$, and $s_{3}$ (one for each basis state) are introduced to describe the resonant current through the uncoupled basis states. These $s$ parameters essentially represent the tunneling amplitudes through the relevant uncoupled basis states in the down- 
stream dot when each is resonant with the $1 s$-like state in the upstream dot, and they are initially assumed to be independent of $B$ field. Using a Fermi's golden-rule argument, which implicitly assumes that the tunnel rates from the emitter and to the collector through the outer barriers are much larger than the interdot tunnel couplings characterized by the $s$ parameters, ${ }^{18}$ we then compute the resonant current for each branch as the square of the sum of eigenvector components $(v)$ multiplied by uncoupled current amplitudes $(s)$. Explicitly, $I_{j}=\left|\sum_{m} v_{m}^{j} s_{m}\right|^{2}$, where $I_{j}$ is the resonant current of the $j$ $=$ (upper, center, lower) branch, and $m=1,2,3$ is the basisstate index [equivalently, $I_{j}=c\left|\left\langle g \mid \psi_{j}\right\rangle\right|^{2}$, where $c$ is a constant, $g$ is the $1 s$-like ground state of the upstream dot, $\psi_{j}$ is the branch state of the downstream dot, and $\left\langle g \mid \psi_{j}\right\rangle$ is an in-plane overlap integral]. Note that when squared, the $s$ parameters give approximately the branch currents far to the left and right of the crossing region where the states are essentially the uncoupled states and the eigenvector components are either zero or one [see, for example, the trends in Fig. 8(e)]. In Ref. 15, we demonstrated that the model successfully explained the coherent mixing which occurred at the $\gamma$ crossing in the spectrum of dot 2 from device II discussed in Sec. V. The reader is referred to this reference for the specific values determined for the $C$ and $s$ parameters at this crossing, however, most importantly for comparison and discussion below, fitting revealed that this crossing was a two-dominant coupling-type crossing with $C_{12} \approx C_{23} \gg C_{13}$.

We now apply our model to the $\tau$ crossing to interpret the energy-level position and branch current behavior shown in Figs. 8(a) and 8(b). The results of fitting are also shown in Figs. 8(c) and 8(d). The fitted coupling energy parameters are (in units of millielectron volt) $C_{13}=-0.88, C_{12}=-0.85$, and $C_{23}=0.13$, revealing that the anticrossing behavior at this crossing is also determined by two-dominant couplings, i.e., in terms of magnitude $C_{13} \approx C_{12} \gg C_{23}$. However, this result differs from the situation for the $\gamma$ crossing in Fig. 5 where we found that $C_{12} \approx C_{23} \gg C_{13} .{ }^{15}$ Concerning the uncoupled current amplitudes, a practical complication in the modeling, is that even if the mixing in the downstream dot were absent, i.e., $C$ parameters all zero, the resonant currents still can vary with $B$ field (as is evident on close examination of the spectra in Fig. 3 away from the immediate crossing regions). In order to account for this in the model, the current amplitudes can be interpolated throughout the crossing region, and a simple linear variation is found to be sufficient in most cases. ${ }^{15}$ For the $\tau$ crossing, the fitted current amplitude parameters are (in units of $\mathrm{pA}^{1 / 2}$ ) $s_{1}=0.391, s_{2}=1.55$, and $s_{3}$ $=0.844$ at $2.24 \mathrm{~T}$, and $s_{1}=0.418, s_{2}=1.37$, and $s_{3}=1.19$ at 3.4 $\mathrm{T}$, with the values in between linearly interpolated.

The eigenvectors for the $\tau$ crossing presented in Fig. 8(e) are also quite different compared to those for the $\gamma$ crossing (see Fig. 3 in Ref. 15). Nonetheless, the clear suppression of the center branch current still arises as a consequence of coherent mixing leading to destructive interference. To understand this, consider the following. For the condition $C_{12}$ $=C_{13}<0, C_{23}=0$, which is close to that for the fitted parameters, the currents at $\Delta B=0 \mathrm{~T}$ (defined as the point where the uncoupled basis levels meet) are given by the following expressions: $I_{\text {upper }}=\frac{1}{4}\left(\sqrt{2} s_{1}-s_{2}-s_{3}\right)^{2}, I_{\text {center }}=\frac{1}{2}\left(s_{2}-s_{3}\right)^{2}$, and $I_{\text {lower }}=\frac{1}{4}\left(\sqrt{2} s_{1}+s_{2}+s_{3}\right)^{2}$. Thus the dark center branch reso- nance at $\Delta B=0$ T occurs when the influence of $s_{1}$ is "zero," and the current will vanish there if $s_{2}$ and $s_{3}$ are comparable in magnitude and of the same sign. Although of the same sign, $s_{2}$ and $s_{3}$ do not quite have the same magnitude and so some small contribution of $s_{1}$ is required to "trim" the current close to zero. This occurs a little to the right of $\Delta B$ $=0 \mathrm{~T}$ consistent with the values of the interpolated $s$ parameters and the eigenvector components near $\Delta B=0 \mathrm{~T}$.

Summarizing this section, our model has revealed that along with the $\gamma$ crossing of Fig. 5, the $\tau$ crossing of Fig. 8 has two-dominant couplings. Nonetheless, the dark center branch resonance near the center of these two three-level crossings actually arises through destructive interference from different conditions, i.e., dark states formed by threelevel mixing do not arise from a unique set of circumstances and are not specific to QDs of a certain ellipticity (as is well demonstrated in Figs. 5 and 8 for two quite different QDs from two different devices). ${ }^{47}$ Clearly, strong suppression of the resonant current in one branch and the associated dark state formation is a genuine and robust effect originating from three-level mixing. Furthermore, the suppression of an otherwise strong resonance due to destructive interference represents an all-electrical QD analog ${ }^{48-52}$ of coherent population trapping in a three-level system of quantum and atom optics when two of the three couplings are dominant. We note that all-optical coherent population trapping has recently been demonstrated in self-assembled InAs QDs. ${ }^{53}$

\section{CONCLUSIONS AND OUTLOOK}

We have described how we measured single-particle (Fock-Darwin-like) energy spectra of high-symmetry QDs in vertical QD molecules by using the $1 s$-like state of one QD to probe states in the adjacent weakly coupled QD in the single-electron resonant-tunneling regime. We also compared the measured spectra to ideal calculated spectra, and explained how we were able to estimate the QD confinement energies. A notable feature of the measured spectra is widespread level mixing and rich anticrossing behavior when single-particle energy levels approach each other. In order to investigate the coherent nature of the mixing at the level crossing regions, two quite general measurement strategies for reliably determining the resonant currents were described and compared. To demonstrate that the results of both strategies are qualitatively the same, and quantitatively very similar, the measurement strategies were applied to a three-level crossing (the $\gamma$ crossing from the spectrum of dot 2 from device II) exhibiting pronounced effects arising from coherent mixing as a test subject. In addition to this crossing, we measured and modeled the mixing at another three-level crossing (the $\tau$ crossing from the spectrum of dot 1 from device I). Both of these three-level crossings show dark state formation in the center branch near the center of the crossing regions due to destructive interference. While fitting of our data revealed that both of these crossings have two-dominant couplings, we demonstrated that the formation of a dark state can arise from more than one set of circumstances. Our observations can help toward the general engineering and understanding of coupling and consequent mixing between 
many quantum levels in coupled QD systems, and the dark state is a useful tool for quantum coherent phenomena in transport involving multiple quantum levels. ${ }^{48-52,54}$

Lastly, a number of directions for future investigation are clear: (i) although the discussion in the second half of this paper has focused on three-level mixing, mixing at two-level and four-level crossings is also of potential interest, and our mixing Hamiltonian model, initially developed for threelevel mixing, can easily be adapted to study these cases. (ii) The strength of the couplings ( $C$ parameters) at any given level crossing could not be altered for the two devices discussed in this work. This is because each of these devices has only a single gate and so the probed QD potential "landscape," i.e., the local nonideal potential felt by the tunneling electrons, could not be altered. An exciting possibility is to alter the strength of the couplings in situ in devices with multiple gates. ${ }^{55,56}$ (iii) A more extensive comparison between the spectra measured in both bias directions from the same device is also attractive because, in principle, this would allow us to build up a "map" of the perturbations in the confinement potentials of both QDs in a device, which could lead to a deeper microscopic understanding of their origin. ${ }^{57}$ An initial attempt to include symmetry-breaking terms to a model ideal elliptical parabolic confinement potential and account for the observed mixing behavior has shown encouraging results provided one restricts the modeling to one or at most two selected level crossings in a given spectrum. ${ }^{15,34}$ (iv) Finally, it would be interesting to attempt to understand multilevel mixing in the regions where double, triple, quadruple,... electron tunneling is accessible, i.e., in the extensive areas, still largely unexplored, below the SET regions in the $V_{\mathrm{SD}}-V_{\mathrm{G}}$ plane evident in Fig. 4(a), for instance, where the electron-electron interactions become important, and to perform inelastic electron-tunneling spectroscopy at even higher bias where longitudinal-optic-phonon emission can occur. ${ }^{58}$

\section{ACKNOWLEDGMENTS}

We thank A. Bezinger, D. Roth, and M. Malloy for assistance with some of the processing, and K. Ono, T. Kodera, T. Hatano, Y. Tokura, M. Stopa, M. Hilke, G. C. Aers, M. Korkusinski, and R. M. Abolfath for useful discussions. Part of this work is supported by NSERC (Discovery Grant No. 208201), Flemish Science Foundation (FWO-VI), Grant-inAid for Scientific Research S (Grant No. 19104007), B (Grant No. 18340081), and by Special Coordination Funds for Promoting Science and Technology, and MEXT. S.T. acknowledges support from QuEST program (BAA-0824).

\footnotetext{
*Corresponding author. FAX: +1-613-950-0202; chris.payette@nrc-cnrc.gc.ca

${ }^{1}$ L. Jacak, P. Hawrylak, and A. Wojs, Quantum Dots (Springer, Berlin, 1998).

${ }^{2}$ T. Chakraborty, Quantum Dots (Elsevier Science, Amsterdam, 1999).

${ }^{3}$ D. Bimberg, M. Grundmann, and N. N. Ledentsov, Quantum Dot Heterostructures (Wiley, Chichester, 1999).

${ }^{4}$ S. M. Reimann and M. Manninen, Rev. Mod. Phys. 74, 1283 (2002).
}

${ }^{5}$ W. G. van der Wiel, S. De Franceschi, J. M. Elzerman, T. Fujisawa, S. Tarucha, and L. P. Kouwenhoven, Rev. Mod. Phys. 75, 1 (2002).

${ }^{6}$ R. Hanson, L. P. Kouwenhoven, J. R. Petta, S. Tarucha, and L. M. Vandersypen, Rev. Mod. Phys. 79, 1217 (2007).

${ }^{7}$ D. Loss and D. P. DiVincenzo, Phys. Rev. A 57, 120 (1998).

${ }^{8}$ V. Fock, Z. Phys. 47, 446 (1928).

${ }^{9}$ C. G. Darwin, Proc. Cambridge Philos. Soc. 27, 86 (1931).

${ }^{10}$ S. Tarucha, D. G. Austing, T. Honda, R. J. van der Hage, and L. P. Kouwenhoven, Phys. Rev. Lett. 77, 3613 (1996).

${ }^{11}$ L. P. Kouwenhoven, T. H. Oosterkamp, M. W. S. Danoesastro, M. Eto, D. G. Austing, T. Honda, and S. Tarucha, Science 278, 1788 (1997).

${ }^{12}$ K. Ono, D. G. Austing, Y. Tokura, and S. Tarucha, Physica B 314, 450 (2002).

${ }^{13}$ T. Fujisawa, D. G. Austing, Y. Tokura, Y. Hirayama, and S. Tarucha, Nature (London) 419, 278 (2002).

${ }^{14}$ Particularly for two-level systems, anticrossing behavior, although not observed in transport measurements in the context of purely electronic single-particle states in QD structures, is prevalent in many different types of low-dimensional, often coupled, semiconductor nanosystems, diversely exemplified in T. H. Oosterkamp, S. F. Godijn, M. J. Uilenreef, Y. V. Nazarov, N. C. van der Vaart, and L. P. Kouwenhoven, Phys. Rev. Lett. 80, 4951 (1998); L. Zimin, S. V. Nair, and Y. Masumoto, ibid. 80, 3105 (1998); H. J. Krenner, M. Sabathil, E. C. Clark, A. Kress, D. Schuh, M. Bichler, G. Abstreiter, and J. J. Finley, ibid. 94, 057402 (2005); S. F. Fischer, G. Apetrii, U. Kunze, D. Schuh, and G. Abstreiter, Nat. Phys. 2, 91 (2006); B. A. Carpenter, E. A. Zibik, M. L. Sadowski, L. R. Wilson, D. M. Whittaker, J. W. Cockburn, M. S. Skolnick, M. Potemski, M. J. Steer, and M. Hopkinson, Phys. Rev. B 74, 161302(R) (2006); C. Ellenberger, T. Ihn, C. Yannouleas, U. Landman, K. Ensslin, D. Driscoll, and A. C. Gossard, Phys. Rev. Lett. 96, 126806 (2006); E. A. Stinaff, M. Scheibner, A. S. Bracker, I. V. Ponomarev, V. L. Korenev, M. E. Ware, M. F. Doty, T. L. Reinecke, and D. Gammon, Science 311, 636 (2006); K. Hennessy, A. Badolato, M. Winger, D. Gerace, M. Atatüre, S. Gulde, S. Fält, E. L. Hu, and A. Imamoglu, Nature (London) 445, 896 (2007); M. Scheibner, M. Yakes, A. S. Bracker, I. V. Ponomarev, M. F. Doty, C. S. Hellberg, L. J. Whitman, T. L. Reinecke, and D. Gammon, Nat. Phys. 4, 291 (2008).

${ }^{15}$ C. Payette, G. Yu, J. A. Gupta, D. G. Austing, S. V. Nair, B. Partoens, S. Amaha, and S. Tarucha, Phys. Rev. Lett. 102, 026808 (2009).

${ }^{16}$ K. Bergmann, H. Theuer, and B. W. Shore, Rev. Mod. Phys. 70, 1003 (1998).

${ }^{17}$ M. O. Scully and M. S. Zubairy, Quantum Optics (Cambridge University Press, England, 2006).

${ }^{18}$ The estimate, based on calculation, of the tunnel coupling energy quoted refers to the energy splitting between corresponding 
states when the ladders of single-particle energy levels for two identical dots are exactly aligned with each other. Using the atomic-orbital-like notation introduced in Sec. III, $\Delta_{S A S}$ would thus refer to the bare tunnel coupling at the $1 s \rightarrow 1 s, 2 p$ $\rightarrow 2 p, \ldots$ resonances, for example. However, as explained more fully in Sec. III, the resonances of interest in this paper involve the ground single-particle state of one dot and a higher-energy single-particle state of the other dot, namely, the $1 s \rightarrow X$ resonances, where $X$ is any single-particle dot state other than $1 s$. The small, though not insignificant, tunnel couplings at these resonances are not determined by $\Delta_{S A S}$ but are related to natural perturbations in the nonideal dot confinement potentials. In Sec. VI, these interdot tunnel couplings are characterized by the $s$ parameters in our model.

${ }^{19}$ D. G. Austing, T. Honda, K. Muraki, Y. Tokura, and S. Tarucha, Physica B 249-251, 206 (1998).

${ }^{20}$ D. G. Austing, T. Honda, Y. Tokura, and S. Tarucha, Jpn. J. Appl. Phys. 34, 1320 (1995).

${ }^{21}$ S. Amaha, C. Payette, J. A. Gupta, T. Hatano, K. Ono, T. Kodera, Y. Tokura, D. G. Austing, and S. Tarucha, Phys. Status Solidi C 5, 174 (2008).

${ }^{22}$ Self-consistent calculations (not shown) reveal that for our triple-barrier double-quantum-well resonant-tunneling structure, the confinement energy in each well along the $z$ axis, $\hbar \omega_{z}$, is $\sim 70 \mathrm{meV}$. This value is ten to fifteen times larger than the lateral confinement energies, $\hbar \omega_{x}$ and $\hbar \omega_{y}$, in typical vertical QDs.

${ }^{23}$ T. K. Rebane, Teor. Eksp. Khim. 5, 3 (1969) [Theor. Exp. Chem. 5, 1 (1972)].

${ }^{24}$ A. V. Madhav and T. Chakraborty, Phys. Rev. B 49, 8163 (1994).

${ }^{25}$ P. A. Maksym, Physica B 249-251, 233 (1998).

${ }^{26}$ D. G. Austing, S. Sasaki, S. Tarucha, S. M. Reimann, M. Koskinen, and M. Manninen, Phys. Rev. B 60, 11514 (1999).

${ }^{27}$ S. Raymond, S. Studenikin, A. Sachrajda, Z. Wasilewski, S. J. Cheng, W. Sheng, P. Hawrylak, A. Babinski, M. Potemski, G. Ortner, and M. Bayer, Phys. Rev. Lett. 92, 187402 (2004).

${ }^{28}$ O. Makarovsky, O. Thomas, A. G. Balanov, L. Eaves, A. Patanè, R. P. Campion, C. T. Foxon, E. E. Vdovin, D. K. Maude, G. Kiesslich, and R. J. Airey, Phys. Rev. Lett. 101, 226807 (2008).

${ }^{29}$ The lateral confinement potentials of vertical QDs, though not infinitely high, are certainly sufficiently high that the employment of the Fock-Darwin spectrum and the elliptical parabolic spectrum for our basic arguments is quite justified provided one does not go too high up in energy (or equivalently let $x$ and $y$ get too large). This certainly holds even for the single-particle energy levels which are $\sim 25 \mathrm{meV}$ up in the probed dot spectra (see Fig. 2).

${ }^{30}$ That the ellipticity of vertical QDs in circular mesas is never exactly unity and varies considerably from device-to-device (typically in the range of $\sim 1.05-2$ ) is well documented for measurements in the few-electron regime. In addition to Refs. 10 and 13, see also P. Matagne, J. P. Leburton, D. G. Austing, and S. Tarucha, Phys. Rev. B 65, 085325 (2002). Natural perturbations in the confinement potentials of vertical QDs caused by local randomness and imperfections are ultimately responsible for this, as well as for the anticrossing behavior in the measured energy spectra.
${ }^{31} \mathrm{~K}$. Ono (unpublished). Single-electron tunneling with longitudinal-optic-phonon emission only influences the measured single-particle spectra above the energy windows shown in Figs. 3(a) and 3(c), and so plays no role in the experiments we describe.

${ }^{32}$ C. Payette, D. G. Austing, G. Yu, J. A. Gupta, S. V. Nair, B. Partoens, S. Amaha, and S. Tarucha, Physica E 40, 1807 (2008).

${ }^{33}$ C. Payette (unpublished).

${ }^{34}$ C. Payette, B. Partoens, G. Yu, J. A. Gupta, D. G. Austing, S. V. Nair, S. Amaha, and S. Tarucha, Appl. Phys. Lett. 94, 222101 (2009).

${ }^{35}$ D. V. Melnikov and J. P. Leburton, Phys. Rev. B 73, 085320 (2006).

${ }^{36}$ K. Ono, D. G. Austing, Y. Tokura, and S. Tarucha, Science 297, 1313 (2002).

${ }^{37}$ L. P. Kouwenhoven, D. G. Austing, and S. Tarucha, Rep. Prog. Phys. 64, 701 (2001).

${ }^{38}$ Thus in each panel of Fig. 3, along the vertical axis corresponding to energy $(E), V_{\mathrm{SD}}$, and $V_{\mathrm{G}}$ are altered such that $\Delta V_{\mathrm{G}} / \Delta V_{\mathrm{SD}}=\lambda$, where $\lambda$ is a constant. Also, from Sec. $\mathrm{V}$ onward, when we use the term differential conductance in connection with strategy A measurements, strictly speaking we are referring to $d I / d E$ but this is simply proportional to $d I / d V_{\mathrm{SD}}$.

${ }^{39}$ The data sets used to build up the parts of spectra shown in Figs. 3(a)-3(c) had no extra spectral features [see, for example, Fig. 2(a) of Ref. 15, for data set related to Fig. 3(c)]. The data set used to build up Fig. 3(d) did have some extra spectral features but it was straightforward to exclude the unwanted extra lines by comparison with the calculated spectrum in Fig. 2(a). Thus the vector-voltage lines employed were well set up for the measurements related to Figs. 3(a)-3(c) but not quite so well set up for the measurement related to Fig. 3(d).

${ }^{40}$ D. G. Austing (unpublished). Although not shown, the energy spectrum of dot 1 from device I (device II) is overall quite similar in general appearance, but not fine detail, to that of dot 2 from the same device (for example, the dot ellipticities are comparable).

${ }^{41}$ M. Pi, A. Emperador, M. Barranco, F. Garcias, K. Muraki, S. Tarucha, and D. G. Austing, Phys. Rev. Lett. 87, 066801 (2001).

${ }^{42}$ D. G. Austing, S. Sasaki, K. Muraki, Y. Tokura, K. Ono, S. Tarucha, M. Barranco, A. Emperador, M. Pi, and F. Garcias, in Advanced Luminescent Materials and Quantum Confinement II, edited by M. Cahay, J. P. Leburton, D. J. Lockwood, S. Bandyopadhyay, N. Koshida, and M. Zacharias (The Electrochemical Society, Pennington, USA, 2002), pp. 13-32.

${ }^{43}$ M. Pi, D. G. Austing, R. Mayol, K. Muraki, S. Sasaki, H. Tamura, and S. Tarucha, in Quantum Dots: New Research, edited by P. A. Ling (Nova Science, New York, USA, 2005), Chap. 1, pp. 1-37.

${ }^{44}$ Also, the position of the $1 s \rightarrow 1 s$ resonance line in the $V_{\mathrm{SD}^{-}} V_{\mathrm{G}}$ plane hardly moves on application of the $B$ field (not shown). This is what we would expect for our measurement scheme. If the upstream and downstream dots are identical the diamagnetic shift of the ground state in both dots would be the same.

${ }^{45}$ The lower edge of the reverse bias SET region in Fig. 4(a) actually weakens and eventually disappears at high bias $\left(V_{\mathrm{SD}}<\right.$ $-50 \mathrm{mV}$ ). The reason for this is not fully understood. However, in the vicinity of the $\gamma$ crossing for each panel in Fig. 6, this edge, if visible, would bisect the region of interest (that bounded by the two dashed lines in the $1.9 \mathrm{~T}$ panel) into two zones. The 
upper zone only is strictly part of the reverse bias SET region highlighted and marked at lower bias in Fig. 4(a). In the lower zone, double-electron tunneling can occur but apparently the additional tunneling processes are very weak as no extra spectral features (resonance lines) are evident in contrast to the region below this zone. Thus inside the extended region of interest consisting of both zones SET dominates, and so our characterization and discussion of the level mixing remains valid. The quarter (three quarters) points defined in the $2.4 \mathrm{~T}$ panel of Fig. 6 lie in the lower (upper) zone. The half points lie close to the boundary between the two zones.

${ }^{46}$ S. M. Huang, Y. Tokura, H. Akimoto, K. Kono, J. J. Lin, S. Tarucha, and K. Ono, Phys. Rev. Lett. 104, 136801 (2010). For weakly coupled vertical QDs and experimental conditions different from those described in this paper, variations in the properties of resonance lines have recently been reported. Also evident in Fig. 1 of this work is the weakening and eventual disappearance of the lower edge of the arc-shaped SET region at high bias described in Ref. 45.

${ }^{47}$ T. Kodera, K. Ono, S. Amaha, Y. Tokura, Y. Arakawa, and S. Tarucha, Phys. Status Solidi C 5, 2854 (2008). Dark state formation is also evident in the data for the three nearly degenerate levels in the third shell close to $0 \mathrm{~T}$ of a vertical QD in another device, although details of the coherent mixing were not presented.
${ }^{48}$ T. Brandes, Phys. Rep. 408, 315 (2005).

${ }^{49}$ A. D. Greentree, J. H. Cole, A. R. Hamilton, and L. C. L. Hollenberg, Phys. Rev. B 70, 235317 (2004).

${ }^{50}$ B. Michaelis, C. Emary, and C. W. J. Beenakker, Europhys. Lett. 73, 677 (2006).

${ }^{51}$ E. del Valle, F. P. Laussy, and C. Tejedor, Europhys. Lett. 80, 57001 (2007).

${ }^{52}$ C. Emary, C. Pöltl, and T. Brandes, Phys. Rev. B 80, 235321 (2009).

${ }^{53}$ X. Xu, B. Sun, P. R. Berman, D. G. Steel, A. S. Bracker, D. Gammon, and L. J. Sham, Nat. Phys. 4, 692 (2008).

${ }^{54}$ M. Korkusinski, I. P. Gimenez, P. Hawrylak, L. Gaudreau, S. A. Studenikin, and A. S. Sachrajda, Phys. Rev. B 75, 115301 (2007).

${ }^{55}$ C. Payette, S. Amaha, T. Hatano, K. Ono, J. A. Gupta, G. C. Aers, D. G. Austing, S. V. Nair, and S. Tarucha, arXiv:0908.4561, Physica E (to be published).

${ }^{56} \mathrm{~S}$. Amaha (unpublished).

${ }^{57}$ O. Makarovsky, A. G. Balanov, L. Eaves, A. Patanè, R. P. Campion, C. T. Foxon, and R. J. Airey, Phys. Rev. B 81, 035323 (2010). In this recent interesting work, randomly distributed ionized donors in double-barrier resonant-tunneling structures are shown to lead to QD-like potential minima.

${ }^{58} \mathrm{~K}$. Ono (unpublished). 